\title{
Sampling and reconstruction by means of weighted inverses
}

\author{
M. Laura Arias ${ }^{1,2} a$ and M. Celeste Gonzalez $z^{1,3} b *$
}

AMS Classification: 94A20, 46C50, 47A58

Keywords: sampling problems, perfect reconstructions, weighted inverses

\begin{abstract}
In this article, we address the problem of reconstructing an element in a Hilbert space from its samples by means of a weighted least square approximation. We show how this problem is linked with the notions of weighted inverses, weighted projections and an angle condition known as compatibility. In addition, we study perfect reconstruction operators and their relationship with the previous problem. Finally, since the reconstructions through these approaches may not be unique, we propose different criteria for choosing an optimal one among all of them.
\end{abstract}

\section{Introduction}

Generalized sampling in separable Hilbert spaces deals with the problem of reconstructing an unknown element $f \in \mathcal{H}$, where $\mathcal{H}$ is a separable Hilbert space, through a collection of samples of $f$. Mathematically, the generalized sampling and reconstruction framework can be described as follows: let $f \in \mathcal{H}$ and $\mathcal{U}, \mathcal{T}$ be two closed subspaces of $\mathcal{H}$ called, respectively, the sampling and reconstruction subspaces. Given a frame $\left\{u_{n}\right\}_{n \in \mathbb{N}}$ for the subspace $\mathcal{U}$ the samples of $f$ are $\left\{\left\langle f, u_{n}\right\rangle\right\}_{n \in \mathbb{N}}$. On the other hand, let $\left\{t_{n}\right\}_{n \in \mathbb{N}}$ be a frame for the subspace $\mathcal{T}$. Then, the problem is to approximate $f$ by a series expansion of the form $\tilde{f}=\sum_{n \in \mathbb{N}} c_{n} t_{n}$ from its samples. In this article we assume that the approximation $\tilde{f}$ is obtained by a bounded linear operator $G: \ell^{2} \rightarrow \mathcal{H}$ with range in $\mathcal{T}$ (in symbols, $G \in \mathcal{L}\left(\ell^{2}, \mathcal{H}\right)$ with

\footnotetext{
${ }^{*}$ The authors were partially supported by CONICET (PIP 11220120100426), FONCYT
} (PICT 2017-0883 and 2014-1776 
$\mathcal{R}(G) \subseteq \mathcal{T})$, called a reconstruction operator. Thus, if $U \in \mathcal{L}\left(\ell^{2}, \mathcal{H}\right)$ is the synthesis operator associated to $\left\{u_{n}\right\}_{n \in \mathbb{N}}$ then the samples of $f$ are $U^{*} f$ and $\tilde{f}=G U^{*} f$. Clearly, in this framework, the reconstructed element is constrained to lie in $\mathcal{T}$. As a consequence, $f$ cannot be perfectly recovered under this scheme if $f \notin \mathcal{T}$. Accordingly, we must select an appropriate reconstruction operator $G_{0} \in \mathcal{L}\left(\ell^{2}, \mathcal{H}\right)$ such that the reconstruction obtained by $G_{0}$ verifies some optimal criterion. Different reconstruction techniques or criteria have been developed during the last decades. In what follows, we summarize some of these criteria:

a) Perfect reconstruction:

$$
G_{0} U^{*} f=f
$$

for all $f \in \mathcal{T}$, i.e., $f$ is perfectly recovered if $f \in \mathcal{T}$. This kind of reconstructions has been studied in [8] and [25], among others works.

b) Consistent reconstruction:

$$
U^{*} G_{0} U^{*} f=U^{*} f
$$

for all $f \in \mathcal{H}$, i.e., the samples of the reconstructed element and those of the original $f$ are equal. This reconstruction condition was introduced by Unser and Aldroubi in [33] and developed later in abstract Hilbert spaces in [24], [26], [18], [5] among others sources.

c) Least squares approximation:

$$
\left\|U^{*} G_{0} d-d\right\|^{2}=\min _{g \in \mathcal{T}}\left\|U^{*} g-d\right\|^{2},
$$

for all $d \in \ell^{2}$. We refer the reader to [8] for this approach. In addition, we recommend [3] and [5] for the particular case in which $d=U^{*} f$.

d) Weighted least squares approximation:

$$
\left\|U^{*} G_{0} d-d\right\|_{A}^{2}=\min _{g \in \mathcal{T}}\left\|U^{*} g-d\right\|_{A}^{2},
$$

for all $d \in \ell^{2}$. Here $A \in \mathcal{L}^{+}\left(\ell^{2}\right)$, i.e., $A$ is a semi-definite positive operator on $\ell^{2}$, and $\|x\|_{A}:=\left\|A^{1 / 2} x\right\|$ for all $x \in \ell^{2}$. Notice that $\|\cdot\|_{A}$ defines a norm if $A$ is an invertible operator. Under this hypothesis, problem $d$ ) is studied in [3]. In the general case, $\|\cdot\|_{A}$ is a semi-norm and so the problem must be studied with different techniques. In [8] this problem is studied for $A=\left(U^{*} U\right)^{\dagger}$. 
The use of weights is a useful strategy when, for example, there is prior information on the original signal that suggests that some parts of the signal to be recovered are more relevant than others. In most applications the operator weight is considered to be an invertible or diagonal operator, however, there are some problems in which the use of a more general weight may be more convenient. For instance, we refer the reader to [9], [10], [11], [34], among other sources, for applications to image processing and some algorithmical results which use non-invertible or non-diagonal weights. Therefore, our main goal in this article is to study problem $d$ ) for a general weight $A \in \mathcal{L}^{+}\left(\ell^{2}\right)$ and its relationship with problem $\left.a\right)$. For this purpose, the notion of weighted inverse introduced by Rao and Mitra in [29] and later extended for operators in Hilbert spaces in [14] plays a fundamental role. This notion which naturally emerges when studying weighted least squares problems, is completely described in [14] in terms of weighted projections and compatibility. An operator $A \in \mathcal{L}^{+}(\mathcal{H})$ and a closed subspace $\mathcal{S}$ of $\mathcal{H}$ are said to be compatible if there exists a projection $Q \in \mathcal{L}(\mathcal{H})$ with range $\mathcal{S}$ which is Hermitian with respect to the semi-inner product $\langle x, y\rangle_{A}:=\langle A x, y\rangle, \forall x, y \in \mathcal{H}$. This concept was defined in [20] and studied later in a series of papers (among them, [14], [17], [21]). We point out that compatibility theory has several applications in splines [16], [7], sampling and approximation theory [3], [18], [15], frame theory [4], and so on. For instance, concerning sampling problems, in [18] compatibility is linked with consistent reconstructions. On the other hand, in [15] the compatibility theory is applied to study in a more general context the approximation problem stated by Sard in [30]. In this article we provide new results regarding sampling problems $a$ ) and $d$ ) by means of the notion of compatibility and weighted inverses. We believe that this general approach will be useful in different applications.

We briefly describe the contents of the paper. In Section 2 we summarize the notions and results about angles between subspaces, frames, weighted projections and weighted inverses that we shall use along this work. Section 3 is devoted to the study of perfect reconstruction operators. Here, the main contributions are Theorem 3.3 where some new characterizations of perfect reconstruction operators are presented, and Proposition 3.5 and Corollary 3.8 where we describe, in two different senses, optimal perfect reconstruction operators. In Section 4 we explore the set of reconstruction operators that satisfy the minimum (1). The first result in this direction is Theorem 4.1 , where the existence of reconstruction operators solving (1) is characterized by means of the compatibility of certain pair $(A, \mathcal{S})$. In addition, the corresponding reconstruction operators are described in terms of weighted projections. The main part of this result follows from [14]. Clearly, since 
the reconstruction obtained by a weighted least squares approximation is sensitive to the operator weight $A$, it would be useful to have a selection criterion for this operator weight. In this sense, we present two results. On one hand, we propose to choose the weight so that the weighted least squares approximation also be perfect. In Theorem 4.6 we consider this scenario and we prove that the existence of such operators is equivalent to a certain compatibility condition, and we express such reconstruction's operators in terms of the projections associated to the compatibility. On the other hand, in Theorem 4.9 we provide conditions on the operator weight so that the previous optimal reconstruction operators also have the smallest quasi-optimality constant (see Definition 3.6 ). Finally, for a fixed weight $A$, the rest of the section is devoted to providing different criteria for choosing an optimal one from the previous reconstruction operators. We refer the reader to Proposition 4.4 and Theorem 4.9 for these results.

\section{Preliminaries}

Throughout $\mathcal{G}, \mathcal{H}$ and $\mathcal{K}$ denote separable complex Hilbert spaces. By $\mathcal{L}(\mathcal{H}, \mathcal{K})$ we denote the space of all bounded linear operators from $\mathcal{H}$ to $\mathcal{K}$. The algebra $\mathcal{L}(\mathcal{H}, \mathcal{H})$ is abbreviated by $\mathcal{L}(\mathcal{H})$. By $\mathcal{L}^{+}(\mathcal{H})$ we denote the cone of positive (semidefinite) operators of $\mathcal{L}(\mathcal{H})$ i.e., $T \in \mathcal{L}^{+}(\mathcal{H})$ if and only if $\langle T x, x\rangle \geq 0$ for all $x \in \mathcal{H}$. Given $T \in \mathcal{L}(\mathcal{H}, \mathcal{K}), \mathcal{R}(T)$ denotes the range or image of $T, \mathcal{N}(T)$ the nullspace of $T, T^{*}$ the adjoint of $T$ and $T^{\dagger}$ the MoorePenrose inverse of $T$. Recall that $T^{\dagger} \in \mathcal{L}(\mathcal{K}, \mathcal{H})$ if and only if $\mathcal{R}(T)$ is closed. We shall denote by $\mathcal{Q}=\left\{Q \in \mathcal{L}(\mathcal{H}): Q=Q^{2}\right\}$ and $\mathcal{P}=\left\{P \in \mathcal{Q}: P=P^{*}\right\}$. Moreover, the symbol $P_{\mathcal{S}}$ stands for the operator in $\mathcal{P}$ with range $\mathcal{S}$. Given $T \in \mathcal{L}(\mathcal{H})$ we write $P_{T}$ to denote $P_{\overline{R(T)}}$. In addition, we denote by $\mathcal{S}+\mathcal{T}$ the direct sum of the subspaces $\mathcal{S}$ and $\mathcal{T}$ and $\mathcal{S} \ominus \mathcal{T}=\mathcal{S} \cap(\mathcal{S} \cap \mathcal{T})^{\perp}$. If $\mathcal{S}+\mathcal{T}=\mathcal{H}$, then $Q_{\mathcal{S} / / \mathcal{T}}$ represents the operator in $\mathcal{Q}$ with range $\mathcal{S}$ and nullspace $\mathcal{T}$.

\subsection{Angles between subspaces}

A notion which is relevant in this paper is that of angle between subspaces. There are many different ways, most of them related, to define the angle between subspaces in a Hilbert space. Here, we shall consider the Friedrichs angle and Dixmier angle (see [28] and [22]). The Friedrichs angle between two closed subspaces $\mathcal{S}$ and $\mathcal{T}$ of $\mathcal{H}$ is the angle $\theta(\mathcal{S}, \mathcal{T}) \in\left[0, \frac{\pi}{2}\right]$ whose cosine 
is defined by

$c(\mathcal{S}, \mathcal{T})=\sup \left\{|\langle\xi, \eta\rangle|: \xi \in \mathcal{S} \cap(\mathcal{S} \cap \mathcal{T})^{\perp}, \eta \in \mathcal{T} \cap(\mathcal{S} \cap \mathcal{T})^{\perp},\|\xi\| \leq 1,\|\eta\| \leq 1\right\}$.

The Dixmier angle between $\mathcal{S}$ and $\mathcal{T}$ is the angle $\theta_{0}(\mathcal{S}, \mathcal{T}) \in\left[0, \frac{\pi}{2}\right]$ whose cosine is defined by

$$
c_{0}(\mathcal{S}, \mathcal{T})=\sup \{|\langle\xi, \eta\rangle|: \xi \in \mathcal{S}, \eta \in \mathcal{T},\|\xi\| \leq 1,\|\eta\| \leq 1\} .
$$

Clearly, if $\mathcal{S} \cap \mathcal{T}=\{0\}$ then both angles coincide.

Another frequently used definition of angle between subspaces is the following: the angle $\theta_{1}(\mathcal{S}, \mathcal{T}) \in\left[0, \frac{\pi}{2}\right]$ between $\mathcal{S}$ and $\mathcal{T}$ is the angle whose cosine is defined by

$$
c_{1}(\mathcal{S}, \mathcal{T})=\inf _{\xi \in \mathcal{S},\|\xi\|=1}\left\|P_{\mathcal{T}} \xi\right\| .
$$

However, it is straightforward that $c_{1}^{2}(\mathcal{S}, \mathcal{T})=1-c_{0}^{2}\left(\mathcal{S}, \mathcal{T}^{\perp}\right)$, see [32].

In the sequel we repeatedly use the following facts about angles between subspaces whose proofs can be found in [28] and [22].

Proposition 2.1. a) Let $\mathcal{S}, \mathcal{T}$ be two closed subspaces of $\mathcal{H}$. Then, $\mathcal{S}+\mathcal{T}$ is a closed subspace if and only if $c(\mathcal{S}, \mathcal{T})<1$. Moreover, $\mathcal{S}+\mathcal{T}$ is a closed subspace if and only if $c_{0}(\mathcal{S}, \mathcal{T})<1$.

b) Let $A, B \in \mathcal{L}(\mathcal{H})$ with closed ranges, then $\mathcal{R}(A B)$ is closed if and only if $\mathcal{R}(B)+\mathcal{N}(A)$ is closed.

Finally, the next result due to Douglas [23] will be used several times along this note:

Theorem 2.2. Let $B \in \mathcal{L}(\mathcal{H}, \mathcal{K})$ and $C \in \mathcal{L}(\mathcal{G}, \mathcal{K})$. The following conditions are equivalent:

a) There exists $D \in \mathcal{L}(\mathcal{G}, \mathcal{H})$ such that $B D=C$.

b) $\mathcal{R}(C) \subseteq \mathcal{R}(B)$.

Moreover, if the conditions of Douglas' theorem hold then $D \in \mathcal{L}(\mathcal{G}, \mathcal{H})$ solves the operator equation $B X=C$ if and only if $D=B^{\dagger} C+Z$ for some $Z \in \mathcal{L}(\mathcal{G}, \mathcal{H})$ with $\mathcal{R}(Z) \subseteq \mathcal{N}(B)$. See, for instance, [7, Lemma 2.1]. 


\section{$2.2 \quad$ Frames}

A fundamental tool for dealing with sampling problems is the theory of frames for Hilbert spaces. Essentially, a frame is an overcomplete system and, as a consequence, they are useful in practical problems where, for example, robustness and noise suppression play a vital role. Here, we introduce some basic facts about frames in Hilbert spaces. For a complete survey on this theory the reader is referred to [35] and [12], among other sources.

Definition 2.3. Let $\mathcal{T}$ be a closed subspace of $\mathcal{H}$. A sequence $\left\{t_{n}\right\}_{n \in \mathbb{N}}$ in $\mathcal{T}$ is called a frame for $\mathcal{T}$ if there exist numbers $A, B>0$ such that, for every $f \in \mathcal{T}$, it holds:

$$
A\|f\|^{2} \leq \sum_{n \in \mathbb{N}}\left|<f, t_{n}>\right|^{2} \leq B\|f\|^{2} .
$$

The main operators associated with a frame are the analysis, synthesis and frame operators. Given a frame $\left\{t_{n}\right\}_{n \in \mathbb{N}}$ for the subspace $\mathcal{T}$, the associated synthesis operator $T: \ell^{2} \rightarrow \mathcal{H}$ is defined by $T c=\sum_{n \in \mathbb{N}} c_{n} t_{n}$. That is to say, $\left\{t_{n}\right\}_{n \in \mathbb{N}}=\left\{T e_{n}\right\}_{n \in \mathbb{N}}$, where $\left\{e_{n}\right\}_{n \in \mathbb{N}}$ is the canonical orthonormal basis in $\ell^{2}$. The adjoint of the synthesis operator is referred to as the analysis operator $T^{*}: \mathcal{H} \rightarrow \ell^{2}$ which is $T^{*} x=\left\{\left\langle x, t_{n}\right\rangle\right\}_{n \in \mathbb{N}}$. By the frame condition, it is straightforward that $T^{*} \in \mathcal{L}\left(\mathcal{H}, \ell^{2}\right)$ and it has closed range. Moreover, $T \in \mathcal{L}\left(\ell^{2}, \mathcal{H}\right)$ and $\mathcal{R}(T)=\mathcal{T}=\overline{\operatorname{span}}\left\{t_{n}\right\}_{n \in \mathbb{N}}$. Finally, the frame operator is $S=T T^{*}$. The sequence $\left\{S^{\dagger} t_{n}\right\}_{n \in \mathbb{N}}$ is called the canonical dual frame of $\left\{t_{n}\right\}_{n \in \mathbb{N}}$, and every $t \in \mathcal{T}$ possesses the frame expansions $t=\sum_{n \in \mathbb{N}}<t, S^{\dagger} t_{n}>t_{n}=\sum_{n \in \mathbb{N}}<t, t_{n}>S^{\dagger} t_{n}$. Observe that the synthesis operator of the dual frame $\left\{S^{\dagger} t_{n}\right\}_{n \in \mathbb{N}}$ is given by $\left(T^{*}\right)^{\dagger}$.

\subsection{Weighted projections and inverses}

In this subsection, we summarize the results concerning weighted projections, compatibility and weighted inverses which we shall apply in the studying of certain sampling and reconstruction problems. Here, $A \in \mathcal{L}^{+}(\mathcal{H})$, $\mathcal{S}, \mathcal{M}$ are closed subspaces of $\mathcal{H}$ and $B \in \mathcal{L}(\mathcal{H})$ is a closed range operator such that $B(\mathcal{M})$ is closed. By $\|\cdot\|_{A}$ we denote the semi-norm induced by $A$, i.e., $\|x\|_{A}=\left\|A^{1 / 2} x\right\|$, for $x \in \mathcal{H}$.

Definitions 2.4. $\quad$ a) We shall say that the pair $(A, \mathcal{S})$ is compatible if there exists $Q \in \mathcal{Q}$ with range $\mathcal{S}$ such that $A Q=Q^{*} A$. Thus, the pair $(A, \mathcal{S})$ is compatible if the following set $\mathcal{P}(A, \mathcal{S})$ is not empty:

$$
\mathcal{P}(A, \mathcal{S}):=\left\{Q \in \mathcal{L}(\mathcal{H}): Q^{2}=Q, \mathcal{R}(Q)=\mathcal{S}, A Q=Q^{*} A\right\} .
$$


In such case, $P_{A, \mathcal{S}}$ denotes the element in $\mathcal{P}(A, \mathcal{S})$ with nullspace $(A \mathcal{S})^{\perp} \ominus \mathcal{N}$, where $\mathcal{N}=\mathcal{N}(A) \cap \mathcal{S}$.

b) An operator $T \in \mathcal{L}(\mathcal{H})$ is called an A-projection into $\mathcal{S}$ if $\mathcal{R}(T) \subseteq \mathcal{S}$ and

$$
\|y-T y\|_{A} \leq\|y-s\|_{A} \text {, for all } y \in \mathcal{H} \text { and } s \in \mathcal{S} \text {. }
$$

From now on, we denote by

$$
\Pi(A, \mathcal{S}):=\{T \in \mathcal{L}(\mathcal{H}): T \text { is an A-projection into } \mathcal{S}\} .
$$

The relationship between these two concepts was studied in [14]. In the next proposition we compile the results about this topic which will be needed in the sequel. For the proof, we refer the reader to [14, Propositions 4.14, 4.17, 4.22 and Corollary 4.12], [17, Theorem 2.6] and [16, Proposition 4.3].

Proposition 2.5. The pair $(A, \mathcal{S})$ is compatible if and only if there exists an $A$-projection into $\mathcal{S}$. In such case, if $\mathcal{N}=\mathcal{N}(A) \cap \mathcal{S}$ then the following properties hold:

a) $\mathcal{P}(A, \mathcal{S}) \subseteq \Pi(A, \mathcal{S})$

b) $\Pi(A, \mathcal{S})=P_{A, \mathcal{S}}+\mathcal{L}(\mathcal{H}, \mathcal{N})$.

c) $\mathcal{P}(A, \mathcal{S})=P_{A, \mathcal{S}}+\{W \in \mathcal{L}(\mathcal{H}): \mathcal{R}(W) \subseteq \mathcal{N}$ and $\mathcal{S} \subseteq \mathcal{N}(W)\}$

d) If $\mathcal{N}=\{0\}$ then $\Pi(A, \mathcal{S})=\mathcal{P}(A, \mathcal{S})=\left\{P_{A, \mathcal{S}}\right\}$.

e) $\left\|P_{A, \mathcal{S}}\right\| \leq\|T\|$, for all $T \in \Pi(A, \mathcal{S})$.

f) $P_{A, \mathcal{S}}=P_{A, \mathcal{S} \ominus \mathcal{N}}+P_{\mathcal{N}}$.

Since the compatibility condition given in Definition 2.4 may be difficult to verify, the following list contains examples and equivalent conditions to this notion. For the proofs of these facts and some examples we recommend $[20]$ and $[21]$.

Lemma 2.6. a) The pair $(A, \mathcal{S})$ is compatible if and only if $c_{0}\left(\mathcal{S}^{\perp}, \overline{A \mathcal{S}}\right)<$ 1 or, equivalently, $\mathcal{H}=\mathcal{S}+(A \mathcal{S})^{\perp}$.

b) If $A=\left(\begin{array}{cc}a & b \\ b^{*} & c\end{array}\right)$ is the matrix representation of $A$ with respect to the Hilbert decomposition $\mathcal{H}=\mathcal{S} \oplus \mathcal{S}^{\perp}$ then, $(A, \mathcal{S})$ is compatible if and only if $\mathcal{R}(b) \subseteq \mathcal{R}(a)$. 
c) If $A$ has closed range, then $(A, \mathcal{S})$ is compatible if and only if $\mathcal{N}(A)+\mathcal{S}$ is closed. In particular, if $\mathcal{H}$ is finite dimensional or $A$ is invertible then $(A, \mathcal{S})$ is compatible.

d) If $A$ is an injective operator, then $(A, \mathcal{S})$ is compatible if and only if $\mathcal{S}^{\perp} \subseteq \mathcal{R}\left(A+\lambda P_{\mathcal{S}^{\perp}}\right)$ for some (and then for any) $\lambda>0$.

Definition 2.7. An operator $G \in \mathcal{L}(\mathcal{H})$ is called an A-inverse of $B$ restricted to $\mathcal{M}$ if $\mathcal{R}(G) \subseteq \mathcal{M}$ and for each $y \in \mathcal{H}$, it holds that

$$
\|y-B G y\|_{A} \leq\|y-B x\|_{A}
$$

for all $x \in \mathcal{M}$. If $\mathcal{M}=\mathcal{H}$ we simply say that $G \in \mathcal{L}(\mathcal{H})$ is an $A$-inverse of $B$.

From now on we denote by

$$
\mathcal{I}_{A, B, \mathcal{M}}=\{G \in \mathcal{L}(\mathcal{H}): G \text { is an } A \text {-inverse of } B \text { restricted to } \mathcal{M}\} .
$$

In the following result we collect some properties of $\mathcal{I}_{A, B, \mathcal{M}}$ :

\section{Proposition 2.8.}

a) $G \in \mathcal{I}_{A, B, \mathcal{M}}$ if and only if $\mathcal{R}(G) \subseteq \mathcal{M}$ and $P_{\mathcal{M}}\left(B^{*} A B G-B^{*} A\right)=0$.

b) $\mathcal{I}_{A, B, \mathcal{M}}$ is not empty if and only if the pair $(A, B(\mathcal{M}))$ is compatible. In such case,

$$
\mathcal{I}_{A, B, \mathcal{M}}=\left\{P_{\mathcal{M}} X: X \in \mathcal{L}(\mathcal{H}), B P_{\mathcal{M}} X \in \Pi(A, B(\mathcal{M}))\right\}
$$

Proof. a) See [14, Remark 5.13].

b) For the first part, the reader is referred to [14, Corollary 5.15]. Hence, let us assume that $\mathcal{I}_{A, B, \mathcal{M}}$ is not empty and let us prove that $\mathcal{I}_{A, B, \mathcal{M}}=$ $\left\{P_{\mathcal{M}} X: B P_{\mathcal{M}} X \in \Pi(A, B(\mathcal{M}))\right\}$. Thus, let $G \in \mathcal{I}_{A, B, \mathcal{M}}$. Then, by item a), $P_{\mathcal{M}} B^{*}\left(A B P_{\mathcal{M}} G-A\right)=0$. So that, $\mathcal{R}\left(A B P_{\mathcal{M}} G-A\right) \subseteq \mathcal{N}\left(P_{\mathcal{M}} B^{*}\right)=$ $B(\mathcal{M})^{\perp}=\mathcal{N}\left(P_{B(\mathcal{M})}\right)$. Then $P_{B(\mathcal{M})}\left(A B P_{\mathcal{M}} G-A\right)=0$ and therefore, by [14, Corollary 4.13] it holds that $B P_{\mathcal{M}} G \in \Pi(A, B(\mathcal{M}))$. Conversely, let $T \in \Pi(A, B(\mathcal{M}))$ and $X \in \mathcal{L}(\mathcal{H})$ such that $B P_{\mathcal{M}} X=T$. By Proposition 2.5, $T=P_{A, B(\mathcal{M})}+Z$, where $Z \in \mathcal{L}(\mathcal{H}, \mathcal{N}(A) \cap B(\mathcal{M}))$. Then $P_{\mathcal{M}}\left(B^{*} A B P_{\mathcal{M}} X-\right.$ $\left.B^{*} A\right)=P_{\mathcal{M}} B^{*} A\left(P_{A, B(\mathcal{M})}+Z-I\right)=P_{\mathcal{M}} B^{*} A\left(P_{A, B(\mathcal{M})}-I\right)=0$. So that, by item $a$ ) it holds that $P_{\mathcal{M}} X \in \mathcal{I}_{A, B, \mathcal{M}}$. 


\section{Perfect reconstruction operators}

We start this section by formulating the sampling problem that we shall treat in this paper. Let $f \in \mathcal{H}$ be the original (unknown) element and let us assume that we are given a sequence of samples $\left\{\left\langle f, u_{j}\right\rangle\right\}_{j \in \mathbb{N}}$ where $\left\{u_{j}\right\}_{j \in \mathbb{N}}$ is a frame for the subspace $\mathcal{U}=\overline{\operatorname{span}}\left\{u_{j}\right\}_{j \in \mathbb{N}}$. We call $\mathcal{U}$ the sampling space. In others words, if $U \in \mathcal{L}\left(\ell^{2}, \mathcal{H}\right)$ is the synthesis operator associated to the frame $\left\{u_{j}\right\}_{j \in \mathbb{N}}$, then the sequence of samples is $U^{*} f$. On the other hand, let $\mathcal{T}$ be a closed subspace of $\mathcal{H}$, called the reconstruction space, and let $\left\{t_{j}\right\}_{j \in \mathbb{N}}$ be a frame of $\mathcal{T}$. Our goal is to approximate $f$ by a series expansion $\tilde{f}=\sum c_{j} t_{j} \in \mathcal{T}$ obtained as $\tilde{f}=G U^{*} f$ for some $G \in \mathcal{L}\left(\ell^{2}, \mathcal{H}\right)$ with $\mathcal{R}(G) \subseteq \mathcal{T}$. The operator $G$ is called a reconstruction operator.

Clearly, if $T \in \mathcal{L}\left(\ell^{2}, \mathcal{H}\right)$ is the synthesis operator associated to the frame $\left\{t_{j}\right\}_{j \in \mathbb{N}}$ we get that $\mathcal{R}(G) \subseteq \mathcal{T}=\mathcal{R}(T)$.

In this section we shall focus on reconstruction operators that are exact on the given reconstruction subspace. More precisely:

Definition 3.1. We shall say that a reconstruction operator $G \in \mathcal{L}\left(\ell^{2}, \mathcal{H}\right)$ is perfect if $G U^{*} g=g$ for all $g \in \mathcal{R}(T)$.

The following characterization of the existence of a perfect reconstruction operator was proven in [8, Theorem 2.2 and Lemma 2.6]. Here we present an alternative proof.

Proposition 3.2. The following assertions are equivalent:

a) There exists a perfect reconstruction operator;

b) $c_{0}\left(\mathcal{R}(T), \mathcal{N}\left(U^{*}\right)\right)<1$.

Proof. $a) \rightarrow b)$. Let $G \in \mathcal{L}\left(\ell^{2}, \mathcal{H}\right)$ be a perfect reconstruction operator. Then, as $\mathcal{R}(G) \subseteq \mathcal{R}(T),\left(G U^{*}\right)^{2} h=G U^{*} h$ for all $h \in \mathcal{H}$. Thus $\left(G U^{*}\right)^{2}=$ $G U^{*}$. Further, as $G U^{*} g=g$ for all $g \in \mathcal{R}(T)$, then $\mathcal{R}(T) \subseteq \mathcal{R}\left(G U^{*}\right) \subseteq$ $\mathcal{R}(G) \subseteq \mathcal{R}(T)$, i.e., $\mathcal{R}\left(G U^{*}\right)=\mathcal{R}(T)$. Thus, as $\mathcal{N}\left(U^{*}\right) \subseteq \mathcal{N}\left(G U^{*}\right)$, we get that $c_{0}\left(\mathcal{R}(T), \mathcal{N}\left(U^{*}\right)\right) \leq c_{0}\left(\mathcal{R}(T), \mathcal{N}\left(G U^{*}\right)\right)<1$, where $c_{0}\left(\mathcal{R}(T), \mathcal{N}\left(G U^{*}\right)\right)<$ 1 because $G U^{*}$ is an idempotent operator with $\mathcal{R}\left(G U^{*}\right)=\mathcal{R}(T)$.

$a) \leftarrow b$ ). If $c_{0}\left(\mathcal{R}(T), \mathcal{N}\left(U^{*}\right)\right)<1$ or, equivalently, $\mathcal{R}(T) \dot{+} \mathcal{N}\left(U^{*}\right)$ is a closed subspace then there exists a closed subspace $\mathcal{S}$ such that $\mathcal{S} \dot{+}$ $\mathcal{N}\left(U^{*}\right)=\mathcal{H}$ and $\mathcal{R}(T) \subseteq \mathcal{S}$. Then, $Q_{\mathcal{S} / / \mathcal{N}\left(U^{*}\right)}$ is well defined and the equation $Q_{\mathcal{S} / / \mathcal{N}\left(U^{*}\right)}=X U^{*}$ has a solution in $\mathcal{L}\left(\ell^{2}, \mathcal{H}\right)$, denoted by $X_{0}$. Define $G=P_{T} X_{0}$. Hence, $\mathcal{R}(G) \subseteq \mathcal{R}(T)$ and $G U^{*} g=P_{T} X_{0} U^{*} g=P_{T} Q_{\mathcal{S} / / \mathcal{N}\left(U^{*}\right)} g=$ $g$ for all $g \in \mathcal{R}(T)$. Therefore $G$ is a perfect reconstruction operator. 
Observe that the existence of perfect reconstruction operators is related to the notion of rich data introduced in reproducing kernel Hilbert spaces by Smale and Zhou [31] and later extended for arbitrary Hilbert spaces by Antezana and Corach in [3]. Given two frames with synthesis operators $F, G$, respectively, $G$ provides rich data with respect to $F$ if $\inf \left\{\left\|G^{*} F z\right\|:\|z\|=\right.$ $\left.1, z \in \mathcal{N}(F)^{\perp}\right\}>0$. Now, by [3, Proposition 3.2], $G$ provides rich data with respect to $F$ if and only if $c_{0}\left(\mathcal{R}(F), \mathcal{R}(G)^{\perp}\right)<1$. Therefore, by Proposition 3.2 , there exists a perfect reconstruction operator for the sampling space $\mathcal{U}$ and the reconstruction space $\mathcal{T}$ if and only if $U$ provides rich data with respect to $T$.

From now on, we denote by $\mathcal{R}_{P}$ the set of perfect reconstruction operators. Our goal is to study this set and to obtain, in some sense explained later, optimal operators in $\mathcal{R}_{P}$. For this purpose, in the following result we list some characterizations of the operators in $\mathcal{R}_{P}$ in terms of idempotent operators, inner inverses and frames. Concerning this last point, notice that if $G \in \mathcal{R}_{P}$ then $\mathcal{R}(G)=\mathcal{T}$, i.e., $G$ is the synthesis operator of some frame for $\mathcal{T}$. In the next result we fully describe those frames.

Theorem 3.3. Let $c_{0}\left(\mathcal{R}(T), \mathcal{N}\left(U^{*}\right)\right)<1$ and $G \in \mathcal{L}\left(\ell^{2}, \mathcal{H}\right)$ be a reconstruction operator. The following conditions are equivalent:

a) $G \in \mathcal{R}_{P}$.

b) $G U^{*}$ is an idempotent operator with $\mathcal{R}\left(G U^{*}\right)=\mathcal{R}(T)$.

c) $G U^{*} G=G$ and $\mathcal{R}(G)=\mathcal{R}(T)$.

d) $U^{*} G$ is an idempotent operator with $\mathcal{N}\left(U^{*} G\right)=\mathcal{N}(G)$ and $\mathcal{R}(G)=$ $\mathcal{R}(T)$.

e) $G$ is the synthesis operator of the canonical dual frame of $\left\{P_{T} U P_{G^{*}} e_{j}\right\}_{j \in \mathbb{N}}$ where $\mathcal{R}\left(G U^{*}\right)=\mathcal{R}(T)$ and $c_{0}\left(\mathcal{R}\left(U G^{*}\right), \mathcal{R}(T)^{\perp}\right)<1$.

Proof. $a) \rightarrow b$ ). The conclusion follows immediately from the proof of $a) \rightarrow$ $b$ ) in Proposition 3.2.

$b) \rightarrow c$ ). Since $G$ is a reconstruction operator then $\mathcal{R}(G) \subseteq \mathcal{R}(T)$. Now, as $\mathcal{R}(T)=\mathcal{R}\left(G U^{*}\right) \subseteq \mathcal{R}(G)$, we get that $\mathcal{R}(G)=\mathcal{R}(T)$. Then, $G U^{*}$ is an idempotent operator with $\mathcal{R}\left(G U^{*}\right)=\mathcal{R}(G)$ and so $G U^{*} G=G$.

$c) \rightarrow d$ ). Clearly, from $G U^{*} G=G$ we obtain that $\left(U^{*} G\right)^{2}=U^{*} G$, i.e., $U^{*} G$ is an idempotent operator. Moreover, $\mathcal{N}\left(U^{*} G\right) \subseteq \mathcal{N}\left(G U^{*} G\right)=$ $\mathcal{N}(G) \subseteq \mathcal{N}\left(U^{*} G\right)$. Hence, $\mathcal{N}\left(U^{*} G\right)=\mathcal{N}(G)$.

$d) \rightarrow e$ ). If $U^{*} G$ is an idempotent operator with $\mathcal{N}\left(U^{*} G\right)=\mathcal{N}(G)$ and $\mathcal{R}(G)=\mathcal{R}(T)$ then $G U^{*}$ is an idempotent operator with $\mathcal{R}\left(G U^{*}\right)=\mathcal{R}(T)$. 
Thus, $G U^{*}=Q_{\mathcal{R}(T) / / \mathcal{M}}$ for some closed subspace $\mathcal{M}$ such that $\mathcal{R}(T) \dot{+}$ $\mathcal{M}=\mathcal{H}$. Hence, $U G^{*}=Q_{\mathcal{M}^{\perp} / / \mathcal{R}(T)^{\perp}}$ and so, $c_{0}\left(\mathcal{R}\left(U G^{*}\right), \mathcal{R}(T)^{\perp}\right)<1$. As a consequence, $\mathcal{R}\left(P_{T} U P_{G^{*}}\right)=\mathcal{R}(T)$, i.e., $\left\{P_{T} U P_{G^{*}} e_{j}\right\}_{j \in \mathbb{N}}$ is a frame for $\mathcal{R}(T)$. Let us prove that $\left(\left(P_{T} U P_{G^{*}}\right)^{*}\right)^{\dagger}=G$. Indeed, $\left(\left(P_{T} U P_{G^{*}}\right)^{*}\right)^{\dagger}=$ $\left(\left(P_{T} U G^{*}\left(G^{*}\right)^{\dagger}\right)^{*}\right)^{\dagger}=\left(\left(P_{T} Q_{\mathcal{M}^{\perp} / / \mathcal{R}(T)^{\perp}}\left(G^{*}\right)^{\dagger}\right)^{*}\right)^{\dagger}=\left(\left(\left(G^{*}\right)^{\dagger}\right)^{*}\right)^{\dagger}=G$.

e) $\rightarrow a)$. First, notice that as $\mathcal{R}\left(G U^{*}\right)=\mathcal{R}(T)$ then $\mathcal{R}(G)=\mathcal{R}(T)$ and, from $c_{0}\left(\mathcal{R}\left(U G^{*}\right), \mathcal{R}(T)^{\perp}\right)<1$, we get that $P_{T} U P_{G^{*}}$ has closed range. Therefore, $\left\{P_{T} U P_{G^{*}} e_{j}\right\}_{j \in \mathbb{N}}$ defines a frame onto its range. Now, since $G$ is the synthesis operator of the canonical dual frame of $\left\{P_{T} U P_{G^{*}} e_{j}\right\}_{j \in \mathbb{N}}$, then $G=\left(\left(P_{T} U P_{G^{*}}\right)^{*}\right)^{\dagger}$ or, equivalently, $\left(G^{*}\right)^{\dagger}=P_{T} U P_{G^{*}}=P_{T} U G^{*}\left(G^{*}\right)^{\dagger}$. Thus, $P_{T}=\left(G^{*}\right)^{\dagger} G^{*}=P_{T} U G^{*}$. From this, we also obtained that $P_{T}=$ $P_{T}\left(U G^{*}\right)^{2}$. Hence, $\mathcal{R}\left(U G^{*}-\left(U G^{*}\right)^{2}\right) \subseteq \mathcal{R}\left(U G^{*}\right) \cap \mathcal{R}(T)^{\perp}=\{0\}$. Therefore, $U G^{*}=\left(U G^{*}\right)^{2}$ and so, $G U^{*}$ is an idempotent operator with $\mathcal{R}\left(G U^{*}\right)=$ $\mathcal{R}(T)$. Therefore, $G U^{*} g=g$ for all $g \in \mathcal{R}(T)$. The proof is complete.

We recommend [8, Theorem 2.2] for some other equivalent conditions to $G \in \mathcal{R}_{P}$. In particular, the equivalence $a \leftrightarrow b$ in the above proposition can also be found (with a different proof) in the mentioned result.

Corollary 3.4. Let $c_{0}\left(\mathcal{R}(T), \mathcal{N}\left(U^{*}\right)\right)<1$. Then,

$$
\left\{G U^{*}: G \in \mathcal{R}_{P}\right\}=\left\{Q \in \mathcal{Q}: \mathcal{R}(Q)=\mathcal{R}(T), \mathcal{N}\left(U^{*}\right) \subseteq \mathcal{N}(Q)\right\} .
$$

Proof. By Proposition 3.2, it follows that if $G$ is a perfect reconstruction operator then $G U^{*} \in \mathcal{Q}, \mathcal{R}\left(G U^{*}\right)=\mathcal{R}(T)$ and, clearly, $\mathcal{N}\left(U^{*}\right) \subseteq \mathcal{N}\left(G U^{*}\right)$. For the other inclusion, if $Q \in \mathcal{Q}, \mathcal{R}(Q)=\mathcal{R}(T)$ and $\mathcal{N}\left(U^{*}\right) \subseteq \mathcal{N}(Q)$, then, by Theorem 2.2, there exists $F \in \mathcal{L}\left(\ell^{2}, \mathcal{H}\right)$ such that $Q=F U^{*}$. Thus, define $G=P_{T} F$. Obviously, $G$ is a reconstruction operator and, since $G U^{*}=Q$, we get that $G \in \mathcal{R}_{P}$.

Notation: Let $c_{0}\left(\mathcal{R}(T), \mathcal{N}\left(U^{*}\right)\right)<1$. Given $Q \in \mathcal{Q}$ with $\mathcal{R}(Q)=\mathcal{R}(T)$ and $\mathcal{N}\left(U^{*}\right) \subseteq \mathcal{N}(Q)$ we denote by

$$
\mathcal{R}_{P}(Q)=\left\{G \in \mathcal{R}_{P}: G U^{*}=Q\right\} .
$$

Proposition 3.5. Let $c_{0}\left(\mathcal{R}(T), \mathcal{N}\left(U^{*}\right)\right)<1$. Then,

$$
\mathcal{R}_{P}(Q)=\left\{Q\left(U^{*}\right)^{\dagger}+P_{T} Z: Z \in \mathcal{L}\left(\ell^{2}, \mathcal{H}\right) \text { with } \mathcal{R}\left(U^{*}\right) \subseteq \mathcal{N}(Z)\right\} .
$$

Furthermore, $G_{Q}:=Q\left(U^{*}\right)^{\dagger} \in \mathcal{R}_{P}(Q)$ is optimal among all the operators in $\mathcal{R}_{P}(Q)$ in the following two senses: 
a) $\left\|\left(U^{*}-G_{Q}^{*}\right) f\right\|=\min \left\{\left\|\left(U^{*}-G^{*}\right) f\right\|: G \in \mathcal{R}_{P}(Q)\right\}$ for all $f \in \mathcal{H}$. Moreover, $G_{Q}$ is the unique $G \in \mathcal{R}_{P}(Q)$ which realizes the minimum.

b) $\left\|U-G_{Q}\right\| \leq\|U-G\|$ for all $G \in \mathcal{R}_{P}(Q)$.

Proof. Let $G \in \mathcal{R}_{P}(Q)$. Then, $G U^{*}=Q$ and, by Theorem 2.2, $G=$ $Q\left(U^{*}\right)^{\dagger}+Z$ for some $Z \in \mathcal{L}\left(\ell^{2}, \mathcal{H}\right)$ with $\mathcal{R}\left(U^{*}\right) \subseteq \mathcal{N}(Z)$. Now, since $\mathcal{R}(G)=\mathcal{R}(T)=\mathcal{R}(Q)$, we get that $G=Q\left(U^{*}\right)^{\dagger}+P_{T} Z$. The other inclusion is trivial.

Let us prove item $a$ ). Consider $G \in \mathcal{R}_{P}(Q)$ then $G=G_{Q}+P_{T} Z$, for some $Z \in \mathcal{L}\left(\ell^{2}, \mathcal{H}\right)$ with $\mathcal{R}\left(U^{*}\right) \subseteq \mathcal{N}(Z)$. Now if $f \in \mathcal{H}$ then $\left\|\left(U^{*}-G^{*}\right) f\right\|^{2}=$ $\left\|\left(U^{*}-G_{Q}^{*}\right) f\right\|^{2}+\left\|Z^{*} P_{T} f\right\|^{2} \geq\left\|\left(U^{*}-G_{Q}^{*}\right) f\right\|^{2}$ because $\mathcal{R}\left(Z^{*}\right) \subseteq \mathcal{R}\left(U^{*}\right)^{\perp}$. Now suppose that there exists $\tilde{G}=G_{Q}+P_{T} \tilde{Z} \in \mathcal{R}_{P}(Q)$ such that $\|\left(U^{*}-\right.$ $\left.\tilde{G}^{*}\right) f\|\leq\|\left(U^{*}-G^{*}\right) f \|$ for all $G \in \mathcal{R}_{P}(Q)$ and for all $f \in \mathcal{H}$. Then, in particular, $\left\|\left(U^{*}-G_{Q}^{*}\right) f\right\|^{2}+\left\|\tilde{Z}^{*} P_{T} f\right\|^{2}=\left\|\left(U^{*}-\tilde{G}^{*}\right) f\right\|^{2} \leq\left\|\left(U^{*}-G_{Q}^{*}\right) f\right\|^{2}$ for all $f \in \mathcal{H}$. So that $\tilde{Z}^{*} P_{T} f=0$ for all $f \in \mathcal{H}$ and therefore $\tilde{Z}^{*} P_{T}=0$. In consequence $\mathcal{R}(\tilde{Z}) \subseteq \mathcal{R}(T)^{\perp}$ and so $\tilde{G}=G_{Q}$.

From item $a$ ) we get that $\left\|U^{*}-G_{Q}^{*}\right\| \leq\left\|U^{*}-G^{*}\right\|$ and so, item $b$ ) holds.

By Theorem 3.3, $G \in \mathcal{R}_{P}(Q)$ is the synthesis operator of the canonical dual frame of $\left\{P_{T} U P_{G^{*}} e_{j}\right\}_{j \in \mathbb{N}}$. Therefore, $\|U-G\|$ can be interpreted as a measure of how well distributed is this frame with respect to the sampling frame $\left\{u_{j}\right\}_{j \in \mathbb{N}}$. In consequence, by the above proposition, the frame associated to $G_{Q}$ is optimal in the sense that it minimizes the distance to $\left\{u_{j}\right\}_{j \in \mathbb{N}}$ among all the frames asociated to the reconstruction operators in $\mathcal{R}_{p}(Q)$. This criteria of optimality was also considered in [2], for the next alternative approach: fixed $Q \in \mathcal{Q}$, the authors in [2] look for all the sampling operators $U$ and all the reconstruction operators $G$ with $\mathcal{R}(G)=\mathcal{R}(Q)$ and $\mathcal{R}(U)=\mathcal{N}(Q)^{\perp}$ such that $G U^{*}=Q$. More precisely, they studied the set $\Gamma_{Q}:=\left\{(G, U): G U^{*}=Q, \mathcal{R}(G)=\mathcal{R}(Q)\right.$ and $\left.\mathcal{R}(U)=\mathcal{N}(Q)^{\perp}\right\}$ and the associated problem $\min _{(G, U) \in \Gamma_{Q}}\|G-U\|^{2}$. The reader is referred to $[2$, Theorems 4.1, 4.3] for these results.

Another criterion of optimality in $\mathcal{R}_{P}$ is proposed in [1] by virtue of the quasi-optimality constant.

Definition 3.6. Let $G \in \mathcal{L}\left(\ell^{2}, \mathcal{H}\right)$ be a reconstruction operator. The quasioptimality constant $\mu=\mu(G)>0$ is the smallest number $\mu$, such that $\left\|f-G U^{*} f\right\| \leq \mu\left\|f-P_{T} f\right\|$, for all $f \in \mathcal{H}$. If $\mu(G)<\infty$ we call $G$ a quasi-optimal operator. 
Trivially, if $G$ is a quasi-optimal operator then it is perfect. Moreover, if $G \in \mathcal{R}_{P}$ then $\mu(G)=\left\|G U^{*}\right\|$, see [8, Corollary 2.5]. Our goal is to describe the elements in $\mathcal{R}_{P}$ with minimal quasi-optimality constant. The key for their description is the next result due to Corach and Maestripieri [16, Proposition 5.2]:

Proposition 3.7. Suppose that $\mathcal{M}$ and $\mathcal{S}$ are two closed subspaces of $\mathcal{H}$ such that $c_{0}(\mathcal{M}, \mathcal{S})<1$. Then,

$$
Q_{0} Q_{0}^{*}=\min \left\{Q Q^{*}: Q \in \mathcal{Q}, \mathcal{R}(Q)=\mathcal{S} \text { and } \mathcal{M} \subseteq \mathcal{N}(Q)\right\}
$$

where $Q_{0}=Q_{\mathcal{S} / / \mathcal{M}+\left(\mathcal{S}^{\perp} \cap \mathcal{M}^{\perp}\right)}$ and the minimum is taken with the Löwner order defined on $\mathcal{L}^{+}(\mathcal{H})$ (i.e., $A \leq B$ if $\langle A x, x\rangle \leq\langle B x, x\rangle, \forall x \in \mathcal{H}$ ); moreover $Q_{0}$ is the unique idempotent satisfying (2).

As immediate consequence of Corollary 3.4 and Proposition 3.7, we present in the following result the expression of the elements in $\mathcal{R}_{P}$ with minimal quasi-optimality constant. To this end, if $c_{0}\left(\mathcal{R}(T), \mathcal{N}\left(U^{*}\right)\right)<1$ we denote by

$$
Q_{0}=Q_{\mathcal{R}(T) / / \mathcal{N}\left(U^{*}\right)+\left(\mathcal{R}(T)+\mathcal{N}\left(U^{*}\right)\right)^{\perp} .}
$$

Corollary 3.8. Let $c_{0}\left(\mathcal{R}(T), \mathcal{N}\left(U^{*}\right)\right)<1$. If $\tilde{G} \in \mathcal{R}_{P}\left(Q_{0}\right)$ then $\tilde{G} U^{*} U \tilde{G}^{*} \leq$ $G U^{*} U G^{*}$ for all $G \in \mathcal{R}_{P}$. In particular, $\left\|\tilde{G} U^{*}\right\| \leq\left\|G U^{*}\right\|$ for all $G \in \mathcal{R}_{P}$.

By Corollaries 3.4 and 3.8, $G_{0}:=Q_{0}\left(U^{*}\right)^{\dagger} \in \mathcal{R}_{P}\left(Q_{0}\right)$ and $\left\|G_{0} U^{*}\right\| \leq$ $\left\|G U^{*}\right\|$, for all $G \in \mathcal{R}_{P}$. That is to say, $G_{0}$ is a perfect reconstruction operator with minimal quasi-optimality constant. We highlight that $G_{0}$ coincides with the perfect reconstruction operator studied in $[8$, Theorems 3.3 and 3.5]. We shall study this particular perfect reconstruction operator with more detail in the next section.

\section{Reconstruction operators by means of restricted weighted inverses}

Given $A \in \mathcal{L}^{+}\left(\ell^{2}\right)$, this section is devoted to study the reconstruction operators $G \in \mathcal{L}\left(\ell^{2}, \mathcal{H}\right), \mathcal{R}(G) \subseteq \mathcal{R}(T)$, such that for all $y \in \ell^{2}$

$$
\left\|y-U^{*} G y\right\|_{A} \leq\left\|y-U^{*} x\right\|_{A}
$$

for all $x \in \mathcal{R}(T)$. Notice that $y \in \ell^{2}$ can be assumed as $U^{*} f+\ell$, i.e., as the original (possible noises) samples. In other words, we propose to minimize 
the distance respect to the semi-norm $\|\cdot\|_{A}$ between the measurements, $U^{*} f+l$, and the samples obtained with a reconstruction $g \in \mathcal{T}$, i.e., $U^{*} g$. Clearly, taking into account Section $2.3, \mathcal{I}_{A, U^{*}, \mathcal{R}(T)}$ is the set of reconstruction operators of problem (3).

In the sequel, since $U^{*}$ and $\mathcal{R}(T)$ are fixed, we simply write $\mathcal{R}^{A}$ instead of $\mathcal{I}_{A, U^{*}, \mathcal{R}(T)}$.

Along this section, we consider that $c\left(\mathcal{R}(T), \mathcal{N}\left(U^{*}\right)\right)<1$ or, equivalently, $\mathcal{R}\left(U^{*} T\right)$ is closed.

Theorem 4.1. Let $A \in \mathcal{L}^{+}\left(\ell^{2}\right)$. Then, $\mathcal{R}^{A}$ is not empty if and only if $\left(A, \mathcal{R}\left(U^{*} T\right)\right)$ is compatible. Moreover, in such case,

$$
\mathcal{R}^{A}=\left\{\left(U^{*} P_{T}\right)^{\dagger} F+P_{T} Z: F \in \Pi\left(A, \mathcal{R}\left(U^{*} T\right)\right), \mathcal{R}(Z) \subseteq \mathcal{N}\left(U^{*} P_{T}\right)\right\} .
$$

This means that given a reconstruction operator $G$, then $G \in \mathcal{R}^{A}$ if and only if $U^{*} G \in \Pi\left(A, \mathcal{R}\left(U^{*} T\right)\right)$. Moreover, if $G \in \mathcal{R}^{A}$ then $G U^{*} \in \Pi\left(U A U^{*}, \mathcal{R}(T)\right)$.

Proof. The first part follows by Proposition 2.8. Let us prove that if $G \in \mathcal{R}^{A}$ then $G U^{*} \in \Pi\left(U A U^{*}, \mathcal{R}(T)\right)$. Hence, consider $G \in \mathcal{R}^{A}$. Then, by (4) and Proposition 2.5, GU* $=\left(U^{*} P_{T}\right)^{\dagger}\left(P_{A, \mathcal{R}\left(U^{*} T\right)}+W\right) U^{*}+P_{T} Z U^{*}$, where $\mathcal{R}(W) \subseteq \mathcal{N}(A) \cap \mathcal{R}\left(U^{*} T\right)$ and $\mathcal{R}(Z) \subseteq \mathcal{N}\left(U^{*} P_{T}\right)$. Then we get that

$$
\begin{aligned}
P_{T} U A U^{*} G U^{*} & =P_{T} U A U^{*}\left(\left(U^{*} P_{T}\right)^{\dagger}\left(P_{A, \mathcal{R}\left(U^{*} T\right)}+W\right) U^{*}+P_{T} Z U^{*}\right) \\
& =P_{T} U A U^{*}\left(U^{*} P_{T}\right)^{\dagger}\left(P_{A, \mathcal{R}\left(U^{*} T\right)}+W\right) U^{*} \\
& =P_{T} U A P_{\mathcal{R}\left(U^{*} T\right)}\left(P_{A, \mathcal{R}\left(U^{*} T\right)}+W\right) U^{*} \\
& =P_{T} U A\left(P_{A, \mathcal{R}\left(U^{*} T\right)}+W\right) U^{*}=P_{T} U A P_{A, \mathcal{R}\left(U^{*} T\right)} U^{*} \\
& =P_{T} U P_{A, \mathcal{R}\left(U^{*} T\right)} A U^{*}=P_{T} U A U^{*} .
\end{aligned}
$$

Then, by [14, Proposition 4.5], it holds that $G U^{*} \in \Pi\left(U A U^{*}, \mathcal{R}(T)\right)$.

Example 4.2. Let $\left\{e_{n}\right\}_{n \in \mathbb{N}}$ be an orthonormal basis of $\mathcal{H}$ and consider the frame sequences $\left\{u_{j}\right\}_{j \in \mathbb{N}}=\left\{e_{2 i-1}, e_{2 i-1}\right\}_{i \in \mathbb{N}, i \neq 3}$ and $\left\{t_{j}\right\}_{j \in \mathbb{N}}=\left\{e_{2 i-1}\right\}_{i \in \mathbb{N}, i \geq 3}$. Let $U$ and $T$ be the synthesis operators associated to $\left\{u_{j}\right\}_{j \in \mathbb{N}}$ and $\left\{t_{j}\right\}_{j \in \mathbb{N}}$, respectively. Note that $\mathcal{R}(T) \cap \mathcal{N}\left(U^{*}\right)=\operatorname{span}\left\{e_{5}\right\} \neq\{0\}$. In addition, $\mathcal{R}(T)+\mathcal{N}\left(U^{*}\right)$ is closed, so that $c\left(\mathcal{R}(T), \mathcal{N}\left(U^{*}\right)\right)<1$. On the other hand, let $\mathcal{S}=\mathcal{R}(T) \cap \mathcal{R}(U)=\overline{\operatorname{span}}\left\{e_{2 i-1}\right\}_{i \in \mathbb{N}, i \geq 4} \subsetneq \mathcal{R}(U), \mathcal{R}(T)$. Take $A \in \mathcal{L}^{+}\left(\ell^{2}\right)$ with closed range and such that $\mathcal{N}(A)=U^{*} \mathcal{S}$. For instance, consider $\tilde{A} \in \mathcal{L}^{+}\left(\ell^{2}\right)$ invertible and then take $A=\left(I-P_{U^{*} \mathcal{S}}\right) \tilde{A}\left(I-P_{U^{*} \mathcal{S}}\right)$. Thus, the pair $\left(A, \mathcal{R}\left(U^{*} T\right)\right)$ is compatible. In fact, $\mathcal{R}\left(U^{*} T\right)+\mathcal{N}(A)=\mathcal{R}\left(U^{*} T\right)+U^{*} \mathcal{S}=$ $\mathcal{R}\left(U^{*} T\right)$ is closed. Then, since $A$ has closed range, by Lemma 2.6, it 
holds that $\left(A, \mathcal{R}\left(U^{*} T\right)\right)$ is compatible. Therefore, by Theorem 4.1 we get that $\mathcal{R}^{A}=\left\{\left(U^{*} P_{T}\right)^{\dagger} F+P_{T} Z, F \in \Pi\left(A, \mathcal{R}\left(U^{*} T\right)\right), \mathcal{R}(Z) \subseteq \mathcal{N}\left(U^{*} P_{T}\right)\right\}$. Now, $\Pi\left(A, \mathcal{R}\left(U^{*} T\right)\right)=P_{A, \mathcal{R}\left(U^{*} T\right)}+\mathcal{L}\left(\ell^{2}, U^{*} \mathcal{S}\right)$, and since $\mathcal{N}\left(P_{A, \mathcal{R}\left(U^{*} T\right)}\right)=$ $\mathcal{R}\left(A U^{*} T\right)^{\perp} \cap\left(U^{*} \mathcal{S}\right)^{\perp}$ then $P_{A, \mathcal{R}\left(U^{*} T\right)}=Q_{\mathcal{R}\left(U^{*} T\right) / / \mathcal{R}\left(A U^{*} T\right)^{\perp} \cap\left(U^{*} \mathcal{S}\right)^{\perp}}$. Therefore, we get that

$$
\begin{array}{r}
\mathcal{R}^{A}=\left\{\left(U^{*} P_{T}\right)^{\dagger}\left(Q_{\mathcal{R}\left(U^{*} T\right) / / \mathcal{R}\left(A U^{*} T\right)^{\perp} \cap\left(U^{*} \mathcal{S}\right)^{\perp}}+W\right)+P_{T} Z:\right. \\
\left.W \in \mathcal{L}\left(\ell^{2}, U^{*} \mathcal{S}\right), \mathcal{R}(Z) \subseteq \mathcal{N}\left(U^{*} P_{T}\right)\right\} .
\end{array}
$$

Corollary 4.3. Let $A \in \mathcal{L}^{+}\left(\ell^{2}\right)$ such that $\mathcal{N}(A) \cap \mathcal{R}\left(U^{*} T\right)=\{0\}$. If $\left(A, \mathcal{R}\left(U^{*} T\right)\right)$ is compatible then

$$
\mathcal{R}^{A}=\left\{T\left(A^{1 / 2} U^{*} T\right)^{\dagger} A^{1 / 2}+P_{T} Z: Z \in \mathcal{L}\left(\ell^{2}, \mathcal{H}\right), \mathcal{R}(Z) \subseteq \mathcal{N}\left(U^{*} P_{T}\right)\right\} .
$$

Proof. If $\mathcal{N}(A) \cap \mathcal{R}\left(U^{*} T\right)=\{0\}$ then $\Pi\left(A, \mathcal{R}\left(U^{*} T\right)\right)=\left\{P_{A, \mathcal{R}\left(U^{*} T\right)}\right\}$.Thus, by Theorem 4.1, it suffices to see that $\left(U^{*} P_{T}\right)^{\dagger} P_{A, \mathcal{R}\left(U^{*} T\right)}=T\left(A^{1 / 2} U^{*} T\right)^{\dagger} A^{1 / 2}$, or, equivalently, that $P_{A, \mathcal{R}\left(U^{*} T\right)}=U^{*} T\left(A^{1 / 2} U^{*} T\right)^{\dagger} A^{1 / 2}$. Let us prove this last equality. A simple computation shows that $Q_{1}:=U^{*} T\left(A^{1 / 2} U^{*} T\right)^{\dagger} A^{1 / 2} \in$ $\mathcal{Q}$ and $A Q_{1}=Q_{1}^{*} A$. Therefore, it only remains to prove that $\mathcal{R}\left(Q_{1}\right)=$ $\mathcal{R}\left(U^{*} T\right)$. Let $y \in \mathcal{R}\left(U^{*} T\right)$. Then $y=U^{*} T x$ for some $x \in \mathcal{H}$ and $Q_{1} y=$ $U^{*} T\left(A^{1 / 2} U^{*} T\right)^{\dagger} A^{1 / 2} y=U^{*} T\left(A^{1 / 2} U^{*} T\right)^{\dagger} A^{1 / 2} U^{*} T x=U^{*} T P_{\mathcal{N}\left(A^{1 / 2} U^{*} T\right)^{\perp}} x=$ $U^{*} T P_{\mathcal{N}\left(U^{*} T\right)^{\perp}} x=y$, where $\mathcal{N}\left(A^{1 / 2} U^{*} T\right)=\mathcal{N}\left(U^{*} T\right)$ since $\mathcal{N}(A) \cap \mathcal{R}\left(U^{*} T\right)=$ $\{0\}$. Thus, $y \in \mathcal{R}\left(Q_{1}\right)$. The other inclusion is trivial. The proof is complete.

In the following result we study, under different scenarios, the behavior of the samples produced by the reconstructions obtained by operators in $\mathcal{R}^{A}$. Before that, we introduce some notation and terminology. Given a compact operator $T \in \mathcal{L}(\mathcal{H}, \mathcal{K})$, it is said that $T$ is in the $p$-Schatten class (which is denoted by $\mathcal{S}_{p}$ ) for some $1 \leq p<\infty$ if $\sum_{k \geq 1} \lambda_{k}(T)^{p}<\infty$, where $\lambda_{k}(T)$ denotes the eigenvalues of $\left(T^{*} T\right)^{1 / 2}$. The class $\mathcal{S}_{p}$ is a normed space with the $p$-Schatten norm $\|T\|_{p}=\left(\sum_{k \geq 1} \lambda_{k}(T)^{p}\right)^{1 / p}$. We refer the readers to [27] for a complete treatment on this class of operators. If $A \in \mathcal{L}^{+}(\mathcal{H})$ and $A^{1 / 2} \in \mathcal{S}_{p}$ for some $1 \leq p<\infty$, the weighted $p$-Schatten norm $\|\cdot\|_{p, A}$ for $T \in \mathcal{L}(\mathcal{H})$ is defined as $\|T\|_{p, A}=\left\|A^{1 / 2} T\right\|_{p}$.

Proposition 4.4. If $\left(A, \mathcal{R}\left(U^{*} T\right)\right)$ is a compatible pair and $\mathcal{N}=\mathcal{N}(A) \cap$ $\mathcal{R}\left(U^{*} T\right)$ then the following assertions hold: 
a) If $f \in \mathcal{H}$ then

$$
\min _{G \in \mathcal{R}^{A}}\left\|U^{*} G U^{*} f\right\|=\left\|P_{A, \mathcal{R}\left(U^{*} T\right) \ominus \mathcal{N}} U^{*} f\right\| .
$$

Moreover, the operators in $\mathcal{R}^{A}$ which satisfy this minimum for all $f \in \mathcal{H}$ are

$$
G=\left(U^{*} P_{T}\right)^{\dagger}\left(P_{A, \mathcal{R}\left(U^{*} T\right) \ominus \mathcal{N}}+V\right)+P_{T} Z,
$$

where $\mathcal{R}\left(U^{*}\right) \subseteq \mathcal{N}(V), \mathcal{R}(V) \subseteq \mathcal{N}$ and $\mathcal{R}(Z) \subseteq \mathcal{N}\left(U^{*} P_{T}\right)$.

b) If $f \in \mathcal{H}$ then

$$
\min _{G \in \mathcal{R}^{A}}\left\|U^{*} f-U^{*} G U^{*} f\right\|=\left\|\left(I-P_{A, \mathcal{R}\left(U^{*} T\right)}\right) U^{*} f\right\| .
$$

Moreover, the operators in $\mathcal{R}^{A}$ which satisfy this minimum for all $f \in \mathcal{H}$ are

$$
G=\left(U^{*} P_{T}\right)^{\dagger}\left(P_{A, \mathcal{R}\left(U^{*} T\right)}+W\right)+P_{T} Z,
$$

where $\mathcal{R}(W) \subseteq \mathcal{N}, \mathcal{R}\left(U^{*}\right) \subseteq \mathcal{N}(W)$ and $\mathcal{R}(Z) \subseteq \mathcal{N}\left(U^{*} P_{T}\right)$.

c) If $A^{1 / 2} \in \mathcal{S}_{p}$, for some $1 \leq p<\infty$, then the reconstruction operators in $\mathcal{R}^{A}$ solve the problem:

$$
\min _{\substack{G \in \mathcal{L}\left(\ell^{2}, \mathcal{H}\right) \\ \mathcal{R}(G) \subseteq \mathcal{R}(T)}}\left\|U^{*} G U^{*}-U^{*}\right\|_{p, A}
$$

Proof.

a) Let $G \in \mathcal{R}^{A}$. Then, by Theorem 4.1 and Proposition 2.5, $G=$ $\left(U^{*} P_{T}\right)^{\dagger} F+P_{T} Z$ for some $Z \in \mathcal{L}\left(\ell^{2}, \mathcal{H}\right)$ with $\mathcal{R}(Z) \subseteq \mathcal{N}\left(U^{*} P_{T}\right)$ and $F=P_{A, \mathcal{R}\left(U^{*} T\right) \ominus \mathcal{N}}+P_{\mathcal{N}}+W$ for some $W \in \mathcal{L}\left(\ell^{2}\right)$ with $\mathcal{R}(W) \subseteq \mathcal{N}$. Hence, if $f \in \mathcal{H}$ then

$$
\begin{aligned}
\left\|U^{*} G U^{*} f\right\|^{2} & =\left\|F U^{*} f\right\|^{2}=\left\|P_{A, \mathcal{R}\left(U^{*} T\right) \ominus \mathcal{N}} U^{*} f\right\|^{2}+\left\|\left(P_{\mathcal{N}}+W\right) U^{*} f\right\|^{2} \\
& \geq\left\|P_{A, \mathcal{R}\left(U^{*} T\right) \ominus \mathcal{N}} U^{*} f\right\|^{2} .
\end{aligned}
$$

Now, $\left\|P_{A, \mathcal{R}\left(U^{*} T\right) \ominus \mathcal{N}} U^{*} f\right\|^{2}=\left\|U^{*}\left(U^{*} P_{T}\right)^{\dagger} P_{A, \mathcal{R}\left(U^{*} T\right) \ominus \mathcal{N}} U^{*} f\right\|^{2}$ and, applying again Theorem 4.1 and Proposition 2.5, $\left(U^{*} P_{T}\right)^{\dagger} P_{A, \mathcal{R}\left(U^{*} T\right) \ominus \mathcal{N}} \in \mathcal{R}^{A}$. Thus, $\min _{G \in \mathcal{R}^{A}}\left\|U^{*} G U^{*} f\right\|=\left\|P_{A, \mathcal{R}\left(U^{*} T\right) \ominus \mathcal{N}} U^{*} f\right\|$. Moreover, by $(5), G \in \mathcal{R}^{A}$ attains this minimum for all $f \in \mathcal{H}$ if and only if $G=\left(U^{*} P_{T}\right)^{\dagger}\left(P_{A, \mathcal{R}\left(U^{*} T\right) \ominus \mathcal{N}}+\right.$ $\left.P_{\mathcal{N}}+W\right)+P_{T} Z$ for some $Z \in \mathcal{L}\left(\ell^{2}, \mathcal{H}\right)$ with $\mathcal{R}(Z) \subseteq \mathcal{N}\left(U^{*} P_{T}\right)$ and some 
$W \in \mathcal{L}\left(\ell^{2}\right)$ with $\mathcal{R}(W) \subseteq \mathcal{N}$ such that $\left(P_{\mathcal{N}}+W\right) U^{*} f=0$ for all $f \in \mathcal{H}$ or, equivalently, $-P_{\mathcal{N}} U^{*}=W U^{*}$. Now, a simple computation shows that $W \in \mathcal{L}\left(\ell^{2}\right)$ satisfies $-P_{\mathcal{N}} U^{*}=W U^{*}$ and $\mathcal{R}(W) \subseteq \mathcal{N}$ if and only if $W=$ $-P_{\mathcal{N}} P_{U^{*}}+V$ for some $V \in \mathcal{L}\left(\ell^{2}\right)$ with $\mathcal{R}(V) \subseteq \mathcal{N}$ and $\mathcal{R}\left(U^{*}\right) \subseteq \mathcal{N}(V)$. The result is proved.

b) Let $G \in \mathcal{R}^{A}$. Then, by Theorem 4.1 and Proposition 2.5, $G=$ $\left(U^{*} P_{T}\right)^{\dagger} F+P_{T} Z$ for some $Z \in \mathcal{L}\left(\ell^{2}, \mathcal{H}\right)$ with $\mathcal{R}(Z) \subseteq \mathcal{N}\left(U^{*} P_{T}\right)$ and $F=P_{A, \mathcal{R}\left(U^{*} T\right)}+W$ for some $W \in \mathcal{L}\left(\ell^{2}\right)$ with $\mathcal{R}(W) \subseteq \mathcal{N}$. Hence, if $f \in \mathcal{H}$ then

$$
\begin{aligned}
\left\|U^{*} f-U^{*} G U^{*} f\right\|^{2} & =\left\|\left(I-U^{*} G\right) U^{*} f\right\|^{2} \\
& =\left\|\left(I-P_{A, \mathcal{R}\left(U^{*} T\right)}\right) U^{*} f\right\|^{2}+\left\|W U^{*} f\right\|^{2} \\
& \geq\left\|\left(I-P_{A, \mathcal{R}\left(U^{*} T\right)}\right) U^{*} f\right\|^{2} .
\end{aligned}
$$

Notice that $\left\|\left(I-P_{A, \mathcal{R}\left(U^{*} T\right)}\right) U^{*} f\right\|^{2}=\left\|U^{*} f-U^{*}\left(\left(U^{*} P_{T}\right)^{\dagger} P_{A, \mathcal{R}\left(U^{*} T\right)}\right) U^{*} f\right\|^{2}$ and $\left(U^{*} P_{T}\right)^{\dagger} P_{A, \mathcal{R}\left(U^{*} T\right)} \in \mathcal{R}^{A}$. Therefore $\min _{G \in \mathcal{R}^{A}}\left\|U^{*} f-U^{*} G U^{*} f\right\|=\|(I-$ $\left.P_{A, \mathcal{R}\left(U^{*} T\right)}\right) U^{*} f \|$. Moreover, by $(6), G \in \mathcal{R}^{A}$ attains this minimum for all $f \in \mathcal{H}$ if and only if $G=\left(U^{*} P_{T}\right)^{\dagger}\left(P_{A, \mathcal{R}\left(U^{*} T\right)}+W\right)+P_{T} Z$ for some $Z \in$ $\mathcal{L}\left(\ell^{2}, \mathcal{H}\right)$ with $\mathcal{R}(Z) \subseteq \mathcal{N}\left(U^{*} P_{T}\right)$ and some $W \in \mathcal{L}\left(\ell^{2}\right)$ with $\mathcal{R}(W) \subseteq \mathcal{N}$ such that $W U^{*}=0$ or, equivalently $\mathcal{R}\left(U^{*}\right) \subseteq \mathcal{N}(W)$. The proof is complete.

c) Let $G \in \mathcal{R}^{A}$. Then, $G=\left(U^{*} P_{T}\right)^{\dagger} F+P_{T} Z \in \mathcal{R}^{A}$, for some $F \in$ $\Pi\left(A, \mathcal{R}\left(U^{*} T\right)\right)$ and $Z \in \mathcal{L}\left(\ell^{2}, \mathcal{H}\right)$ with $\mathcal{R}(Z) \subseteq \mathcal{N}\left(U^{*} P_{T}\right)$. In addition, by Proposition 2.5, $F=P_{A, \mathcal{R}\left(U^{*} T\right)}+W$, for some $W \in \mathcal{L}\left(\ell^{2}\right)$ with $\mathcal{R}(W) \subseteq$ $\mathcal{N}(A) \cap \mathcal{R}\left(U^{*} T\right)$. Therefore,

$$
\begin{aligned}
& P_{T} U A\left(U^{*} P_{T} G U^{*}-U^{*}\right)=P_{T} U A\left[U^{*} P_{T}\left(\left(U^{*} P_{T}\right)^{\dagger} F+P_{T} Z\right) U^{*}-U^{*}\right] \\
= & P_{T} U A\left[F U^{*}-U^{*}\right]= \\
= & P_{T} U A F U^{*}-P_{T} U A U^{*}=P_{T} U A\left(P_{A, \mathcal{R}\left(U^{*} T\right)}+W\right) U^{*}-P_{T} U A U^{*} \\
= & P_{T} U P_{A, \mathcal{R}\left(U^{*} T\right)}^{*} A U^{*}-P_{T} U A U^{*}=0 .
\end{aligned}
$$

Then, by [13, Theorem 4.3 and Corollary 3.3], G solves $\min _{X \in \mathcal{L}\left(\ell^{2}, \mathcal{H}\right)} \| U^{*} P_{T} X U^{*}-$ $U^{*} \|_{p, A}$. Therefore, $G$ solves the desired problem.

For a treatment of the weighted least square solutions of the operator equation $D X B-C=0$ considering the seminorm $\|\cdot\|_{p, A}$, the reader is referred to [13].

Clearly, the choice of $A$ affects the reconstruction obtained by an operator in $\mathcal{R}^{A}$. Therefore, it would be desirable to have a criterion for selection of the operator weight $A$. Here, we propose two criteria. In the first one 
(Theorem 4.6), we propose to choose $A$ so that the weighted least square reconstruction also be perfect, i.e., $\mathcal{R}^{A} \cap \mathcal{R}_{P}$ be not empty. In the second one (Theorem 4.9), we suggest to select the weight $A$ such that there exists a reconstruction operator in $\mathcal{R}^{A} \cap \mathcal{R}_{P}$ with smallest quasi-optimality constant. Our first criterion is inspired in Sard's work [30]. In the following remark we summarize Sard's approach and some of his main results.

Remark 4.5. The approach proposed in [30] is the following:

Let $\mu$ be a Lebesgue-Stieljes measure on $\mathbb{R}, \mathcal{H}$ be the Hilbert space $\mathcal{L}^{2}(\mu)$ and let $(\Omega, \mathcal{F}, \mathbb{P})$ be a probability space. Given $x+\delta x \in \mathcal{H}$, where $\delta x$ is the error, then $x$ is approximate by $T(x+\delta x)$ where $T \in \mathcal{L}(\mathcal{H})$. Such operator $T$ is called a process. It is assumed that $\delta x: \mathbb{R} \times \Omega \rightarrow \mathcal{H}$ is a $\mu \times \mathbb{P}$-measurable function such that:

a) for almost every $t \in \mathbb{R}, E(\delta x(t, \cdot))=0$,

b) for almost every $\omega \in \Omega, \delta x(\cdot, \omega) \in \mathcal{H}$,

c) $E\left(\|\delta x\|^{2}\right)=\int_{\Omega} \int_{\mathbb{R}}|\delta x(\omega, t)|^{2} d \mu d \mathbb{P}(w)<\infty$

The variance $V$ of $\delta x$ is the operator given by $V x=E(\langle x, \delta x\rangle \delta x)$. It holds that $V \in \mathcal{L}^{+}(\mathcal{H})$ and it is a trace operator.

Fixed a closed subspace $\mathcal{M}$ of $\mathcal{H}$ the following two processes are studied in [30]:

- Approximation process: Let $\Xi=\{T \in \mathcal{L}(\mathcal{H}): E(T(x+\delta x))=$ $x \forall x \in \mathcal{M}\}$. Hence, $T \in \Xi$ is called an approximation process over $\mathcal{M}$ if $E\|T \delta x\|^{2} \leq E\|U \delta x\|^{2}$ for all $U \in \Xi$. In [30, Theorem 1], it is proven that $T \in \mathcal{L}(\mathcal{H})$ is an approximation process if and only if $\mathcal{R}\left(V T^{*}\right) \subseteq \mathcal{M}$ and $T x=x$ for all $x \in \mathcal{M}$. This last condition means that if $T$ is an approximation process then the elements of $\mathcal{M}$ are perfectly recovered by $T$. However, since in Sard's scenario $\mathcal{R}(T)$ is not assumed to be included in $\mathcal{M}$, then this approach differs from our perfect reconstruction definition. Later, in [15], the notion of approximation process is extended for an arbitrary $V \in \mathcal{L}(\mathcal{H})^{+}$(i.e. $V$ is not necessarily a trace operator), and it is shown the relationship between the approximation processes over $\mathcal{M}$ and the elements of $\mathcal{P}\left(V, \mathcal{M}^{\perp}\right)$.

- Least square process: Let $A \in \mathcal{L}^{+}(\mathcal{H})$. A least square process is an operator $T \in \mathcal{L}(\mathcal{H})$ such that $\mathcal{R}(T) \subseteq \mathcal{M}$ and for each $f \in \mathcal{H}$, $\|f-T f\|_{A}=\min _{m \in \mathcal{M}}\|f-m\|_{A}$. This kind of processes is studied in [30] under the extra condition $\mathcal{M} \subseteq \mathcal{R}\left(P_{\mathcal{M}} A P_{\mathcal{M}}\right)$ (proper condition), 
and in [15] for the general case. Again, in this last work it is shown the relationship between this sort of processes and the compatibility theory.

Clearly, the choice of $A$ affects the approximation $T(x+\delta x)$ by a least square process T. In [30], it is proposed to choose the weight $A$ such that the least square process be also an approximation process. In the context of [30], it is proven that such a process exits if and only if $V A(\mathcal{M}) \subseteq \mathcal{M}$. For the general case, we recommend [15, Theorem 4.6].

Taking into account Sard's criterion for selection of the weight $A$, in the following result we characterize the operators in $\mathcal{R}^{A} \cap \mathcal{R}_{P}$.

Theorem 4.6. Let $A \in \mathcal{L}^{+}\left(\ell^{2}\right)$. Then, $\mathcal{R}^{A} \cap \mathcal{R}_{P}$ is not empty if and only if $\left(A, \mathcal{R}\left(U^{*} T\right)\right)$ is compatible and $\mathcal{R}(T) \cap \mathcal{N}\left(U^{*}\right)=\{0\}$. Moreover, in such case,

$$
\mathcal{R}^{A} \cap \mathcal{R}_{P}=\left\{\left(U^{*} P_{T}\right)^{\dagger} Q: Q \in \mathcal{P}\left(A, \mathcal{R}\left(U^{*} T\right)\right)\right\} .
$$

That is, $G \in \mathcal{R}^{A} \cap \mathcal{R}_{P}$ if and only if $U^{*} G \in \mathcal{P}\left(A, \mathcal{R}\left(U^{*} T\right)\right)$. Moreover, if $G \in \mathcal{R}^{A} \cap \mathcal{R}_{P}$ then $G U^{*} \in \mathcal{P}\left(U A U^{*}, \mathcal{R}(T)\right)$.

Proof. Clearly, by Proposition 3.2 and Theorem 4.1, if $\mathcal{R}^{A} \cap \mathcal{R}_{P}$ is not empty then $\left(A, \mathcal{R}\left(U^{*} T\right)\right)$ is compatible and $\mathcal{R}(T) \cap \mathcal{N}\left(U^{*}\right)=\{0\}$. Conversely, suppose that $\left(A, \mathcal{R}\left(U^{*} T\right)\right)$ is compatible and $\mathcal{R}(T) \cap \mathcal{N}\left(U^{*}\right)=\{0\}$. Then $\mathcal{N}\left(U^{*} P_{T}\right)=\mathcal{R}(T)^{\perp}$ and so, by Theorem 4.1 , the set $\mathcal{R}^{A}$ is $\left\{\left(U^{*} P_{T}\right)^{\dagger} F\right.$ : $\left.F \in \Pi\left(A, \mathcal{R}\left(U^{*} T\right)\right)\right\}$. Hence, let us take $F=Q \in \mathcal{P}\left(A, \mathcal{R}\left(U^{*} T\right)\right) \subseteq$ $\Pi\left(A, \mathcal{R}\left(U^{*} T\right)\right)$ and let us prove that $\left(U^{*} P_{T}\right)^{\dagger} Q \in \mathcal{R}_{p}$. For this, write $Q=P_{A, \mathcal{R}\left(U^{*} T\right)}+Z$, where $Z \in \mathcal{L}\left(\ell^{2}\right)$ satisfies that $\mathcal{R}(Z) \subseteq \mathcal{N}(A) \cap \mathcal{R}\left(U^{*} T\right)$ and $\mathcal{R}\left(U^{*} T\right) \subseteq \mathcal{N}(Z)$ (see Proposition 2.5). Then

$$
\begin{aligned}
\left(\left(U^{*} P_{T}\right)^{\dagger} Q U^{*}\right)^{2} & =\left(U^{*} P_{T}\right)^{\dagger}\left(P_{A, \mathcal{R}\left(U^{*} T\right)}+Z\right) U^{*} P_{T}\left(U^{*} P_{T}\right)^{\dagger} Q U^{*} \\
& =\left(U^{*} P_{T}\right)^{\dagger} P_{A, \mathcal{R}\left(U^{*} T\right)} U^{*} P_{T}\left(U^{*} P_{T}\right)^{\dagger} Q U^{*} \\
& =\left(U^{*} P_{T}\right)^{\dagger} U^{*} P_{T}\left(U^{*} P_{T}\right)^{\dagger} Q U^{*}=\left(U^{*} P_{T}\right)^{\dagger} Q U^{*}
\end{aligned}
$$

In addition, it is clear that $\mathcal{R}\left(\left(U^{*} P_{T}\right)^{\dagger} Q U^{*}\right) \subseteq \mathcal{R}(T)$. Now, if $x \in \mathcal{R}(T)$ then $x=P_{T} x$ and $\left(U^{*} P_{T}\right)^{\dagger} Q U^{*} P_{T} x=\left(U^{*} P_{T}\right)^{\dagger} P_{A, \mathcal{R}\left(U^{*} T\right)} U^{*} P_{T} x=P_{T} x=$ $x$. In consequence, $\mathcal{R}\left(\left(U^{*} P_{T}\right)^{\dagger} Q\right)=\mathcal{R}(T)$ and so $\left(U^{*} P_{T}\right)^{\dagger} Q \in \mathcal{R}^{A} \cap \mathcal{R}_{P}$. Therefore $\mathcal{R}^{A} \cap \mathcal{R}_{P}$ is not empty and every operator of the form $\left(U^{*} P_{T}\right)^{\dagger} Q$ with $Q \in \mathcal{P}\left(A, \mathcal{R}\left(U^{*} T\right)\right)$ is in $\mathcal{R}^{A} \cap \mathcal{R}_{P}$. Let us prove the remaining inclusion. Let $G \in \mathcal{R}^{A} \cap \mathcal{R}_{P}$. Then, $G=\left(U^{*} P_{T}\right)^{\dagger} F \in \mathcal{R}^{A} \cap \mathcal{R}_{P}$, for some $F \in$ $\Pi\left(A, \mathcal{R}\left(U^{*} T\right)\right)$. We claim that $F \in \mathcal{P}\left(A, \mathcal{R}\left(U^{*} T\right)\right)$. In fact, as $G \in \mathcal{R}_{P}$ then 
$G U^{*}$ is an idempotent onto $\mathcal{R}(T)$. So, if $x \in \mathcal{R}(T)$ and $F=P_{A, \mathcal{R}\left(U^{*} T\right)}+Z$ where $\mathcal{R}(Z) \subseteq \mathcal{R}\left(U^{*} T\right) \cap \mathcal{N}(A)$ (see Proposition 2.5) then

$$
\begin{aligned}
& x=P_{T} x=G U^{*} P_{T} x=\left(U^{*} P_{T}\right)^{\dagger}\left(P_{A, \mathcal{R}\left(U^{*} T\right)}+Z\right) U^{*} P_{T} x \\
& =P_{\mathcal{N}\left(U^{*} P_{T}\right)^{\perp}} x+\left(U^{*} P_{T}\right)^{\dagger} Z U^{*} P_{T} x=P_{T} x+\left(U^{*} P_{T}\right)^{\dagger} Z U^{*} P_{T} x \\
& =x+\left(U^{*} P_{T}\right)^{\dagger} Z U^{*} P_{T} x \text {. }
\end{aligned}
$$

Thus $\left(U^{*} P_{T}\right)^{\dagger} Z U^{*} P_{T} x=0$ and so $Z U^{*} P_{T} x \in \mathcal{R}\left(U^{*} T\right) \cap \mathcal{R}\left(U^{*} T\right)^{\perp}=\{0\}$. Then $Z U^{*} P_{T} x=0$ and therefore $\mathcal{R}\left(U^{*} T\right) \subseteq \mathcal{N}(Z)$. So that, by Proposition 2.5, $F \in \mathcal{P}\left(A, \mathcal{R}\left(U^{*} T\right)\right)$ and the assertion follows.

Now, let us prove that if $G \in \mathcal{R}^{A} \cap \mathcal{R}_{P}$ then $G U^{*} \in \mathcal{P}\left(U A U^{*}, \mathcal{R}(T)\right)$. Thus, assume that $G \in \mathcal{R}^{A} \cap \mathcal{R}_{P}$. Since $G \in \mathcal{R}_{P}$ we know that $G U^{*}$ is an idempotent operator with $\mathcal{R}\left(G U^{*}\right)=\mathcal{R}(T)$, i.e., it only remains to show that $U A U^{*} G U^{*}$ is selfadjoint. Now, from (7), $G=\left(U^{*} P_{T}\right)^{\dagger} Q$ for some $Q \in$ $\mathcal{P}\left(A, \mathcal{R}\left(U^{*} T\right)\right)$. So, $U A U^{*} G U^{*}=U A U^{*} P_{T} G U^{*}=U A U^{*} P_{T}\left(U^{*} P_{T}\right)^{\dagger} Q U^{*}=$ $U A Q U^{*}$, which is a selfadjoint operator since $Q \in \mathcal{P}\left(A, \mathcal{R}\left(U^{*} T\right)\right)$.

Example 4.7. Let $\left\{e_{n}\right\}_{n \in \mathbb{N}}$ be an orthonormal basis of $\mathcal{H}$ and consider the frame sequences $\left\{u_{j}\right\}_{j \in \mathbb{N}}=\left\{e_{2 i-1}, e_{2 i-1}\right\}_{i \in \mathbb{N}, i \neq 3}$ and $\left\{t_{j}\right\}_{j \in \mathbb{N}}=\left\{e_{3}+\right.$ $\left.e_{5}, e_{2 i-1}\right\}_{i \in \mathbb{N}, i \geq 4}$. Let $U$ and $T$ be the synthesis operators associated to $\left\{u_{j}\right\}_{j \in \mathbb{N}}$ and $\left\{t_{j}\right\}_{j \in \mathbb{N}}$, respectively. Notice that $\mathcal{R}(T) \cap \mathcal{N}\left(U^{*}\right)=\{0\}$ and $\mathcal{R}(T)+$ $\mathcal{N}\left(U^{*}\right)$ is closed, i.e., $c_{0}\left(\mathcal{R}(T), \mathcal{N}\left(U^{*}\right)\right)<1$. Let $\mathcal{S}:=\mathcal{R}(T) \cap \mathcal{R}(U) \subsetneq$ $\mathcal{R}(T), \mathcal{R}(U)$ (observe that $\mathcal{S} \neq\{0\}$ ) and take $A \in \mathcal{L}^{+}\left(\ell^{2}\right)$ with closed range such that $\mathcal{N}(A)=U^{*} \mathcal{S}$. Again, as in Example 4.2, we can choose $A=\left(I-P_{U^{*} \mathcal{S}}\right) \tilde{A}\left(I-P_{U^{*} \mathcal{S}}\right)$, with $\tilde{A} \in \mathcal{L}^{+}\left(\ell^{2}\right)$ invertible. Observe that the pair $\left(A, \mathcal{R}\left(U^{*} T\right)\right)$ is compatible because $\mathcal{R}\left(U^{*} T\right)+\mathcal{N}(A)=\mathcal{R}\left(U^{*} T\right)+$ $U^{*} \mathcal{S}=\mathcal{R}\left(U^{*} T\right)$ (see Lemma 2.6). Hence, if $Q \in \mathcal{P}\left(A, \mathcal{R}\left(U^{*} T\right)\right.$ ) then $Q=P_{A, \mathcal{R}\left(U^{*} T\right)}+W$, where $W \in \mathcal{L}\left(\ell^{2}\right), \mathcal{R}(W) \subseteq U^{*} \mathcal{S}$ and $\mathcal{R}\left(U^{*} T\right) \subseteq$ $\mathcal{N}(W)$. Now, since $\mathcal{N}\left(P_{A, \mathcal{R}\left(U^{*} T\right)}\right)=\mathcal{R}\left(A U^{*} T\right)^{\perp} \cap\left(\mathcal{R}\left(U^{*} T\right) \cap U^{*} \mathcal{S}\right)^{\perp}=$ $\mathcal{R}\left(A U^{*} T\right)^{\perp} \cap\left(U^{*} \mathcal{S}\right)^{\perp}$, we get that $P_{A, \mathcal{R}\left(U^{*} T\right)}=Q_{\mathcal{R}\left(U^{*} T\right) / / \mathcal{R}\left(A U^{*} T\right)^{\perp} \cap\left(U^{*} \mathcal{S}\right)^{\perp}}$. Therefore,

$$
\begin{array}{r}
\mathcal{R}^{A} \cap \mathcal{R}_{P}=\left\{\left(U^{*} P_{T}\right)^{\dagger}\left(Q_{\mathcal{R}\left(U^{*} T\right) / / \mathcal{R}\left(A U^{*} T\right)^{\perp} \cap\left(U^{*} \mathcal{S}\right)^{\perp}}+W\right): W \in \mathcal{L}\left(\ell^{2}\right),\right. \\
\left.\mathcal{R}(W) \subseteq U^{*} \mathcal{S}, \mathcal{R}\left(U^{*} T\right) \subseteq \mathcal{N}(W)\right\} .
\end{array}
$$

Remark 4.8. If in Theorem 4.6 we take $A=I$ then the set $\mathcal{R}^{I} \cap \mathcal{R}_{P}$ has a unique element. Several representations of this operator are known (see [3, Proposition 3.4] and [8, Theorem 3.1]). From Theorem 4.6 it holds that $\mathcal{R}^{I} \cap \mathcal{R}_{P}=\left\{\left(U^{*} P_{T}\right)^{\dagger}\right\}$. 
In the next result we obtain, for certain $A \in \mathcal{L}^{+}\left(\ell^{2}\right)$, the elements in $\mathcal{R}^{A} \cap$ $\mathcal{R}_{P}$ with the smallest quasi-optimality constant among all the reconstruction operators in $\mathcal{R}^{A} \cap \mathcal{R}_{P}$.

Theorem 4.9. Let $c_{0}\left(\mathcal{R}(T), \mathcal{N}\left(U^{*}\right)\right)<1$ and $\left(A, \mathcal{R}\left(U^{*} T\right)\right)$ be compatible. If $\mathcal{N}\left(A U^{*}\right) \cap \mathcal{R}(T) \subseteq \mathcal{R}(U)$ then there exists $G_{1} \in \mathcal{R}^{A} \cap \mathcal{R}_{P}$ such that $G_{1} U^{*}=P_{U A U^{*}, R(T)}$. Therefore, $\left\|G_{1} U^{*}\right\| \leq\left\|G U^{*}\right\|$ for all $G \in \mathcal{R}^{A}$.

Before we prove Theorem 4.9, we present the following

Lemma 4.10. If $\left(A, \mathcal{R}\left(U^{*} T\right)\right)$ is compatible then there exists a closed subspace $\mathcal{M}$ of $\mathcal{H}$ such that $\mathcal{M}+\mathcal{N}(U)=\mathcal{H}$ and $\mathcal{R}\left(A U^{*} T\right) \subseteq \mathcal{M}$.

Proof. By [21, Theorem 2.15], the pair $\left(A, \mathcal{R}\left(U^{*} T\right)\right)$ is compatible if and only if $c_{0}\left(\overline{\mathcal{R}\left(A U^{*} T\right)}, \mathcal{R}\left(U^{*} T\right)^{\perp}\right)<1$. Then, since $\mathcal{N}(U) \subseteq \mathcal{R}\left(U^{*} T\right)^{\perp}$, it holds that $c_{0}\left(\overline{\mathcal{R}\left(A U^{*} T\right)}, \mathcal{N}(U)\right)<1$. Then $\overline{\mathcal{R}\left(A U^{*} T\right)} \dot{+} \mathcal{N}(U)$ is closed. Thus, for instance, $\mathcal{M}=\overline{\mathcal{R}\left(A U^{*} T\right)}+\left(\overline{\mathcal{R}\left(A U^{*} T\right)}+\mathcal{N}(U)\right)^{\perp}$ verifies the result.

Proof of Theorem 4.9. As $c_{0}\left(\mathcal{R}(T), \mathcal{N}\left(U^{*}\right)\right)<1$ and $\left(A, \mathcal{R}\left(U^{*} T\right)\right)$ is compatible then, by Theorem $4.6, \mathcal{R}^{A} \cap \mathcal{R}_{P}$ is not empty and $\left(U A U^{*}, \mathcal{R}(T)\right)$ is compatible. Moreover, observe that as $\mathcal{N}\left(A U^{*}\right) \cap \mathcal{R}(T) \subseteq \mathcal{R}(U)$ then $\mathcal{R}\left(P_{U A U^{*}, \mathcal{R}(T)}^{*}\right)=\mathcal{R}(T) \cap \mathcal{N}\left(A U^{*}\right)+\overline{U A U^{*}(\mathcal{R}(T))} \subseteq \mathcal{R}(U)$. Thus, by Theorem 2.2, there exists $F \in \mathcal{L}(\mathcal{H})$ such that $U F=P_{U A U^{*}, \mathcal{R}(T)}^{*}$ or, equivalently $F^{*} U^{*}=P_{U A U^{*}, \mathcal{R}(T)}$. Moreover, by Lemma 4.10, there exists a closed subspace $\mathcal{M}$ of $\mathcal{H}$ such that $\mathcal{M}+\mathcal{N}(U)=\mathcal{H}$ and $\mathcal{R}\left(A U^{*} T\right) \subseteq \mathcal{M}$. Hence, define $G_{1}:=P_{T} F^{*} Q_{\mathcal{M} / / \mathcal{N}(U)}^{*}$. We claim that $G_{1} \in \mathcal{R}^{A} \cap \mathcal{R}_{P}$ and $G_{1} U^{*}=$ $P_{U A U^{*}, \mathcal{R}(T)}$. Trivially, $G_{1} U^{*}=P_{T} F^{*} U^{*}=P_{T} P_{U A U^{*}, \mathcal{R}(T)}=P_{U A U^{*}, \mathcal{R}(T)}$. Moreover, as $G_{1} U^{*}=P_{U A U^{*}, \mathcal{R}(T)}$ and $R\left(G_{1}\right) \subseteq \mathcal{R}(T)$, then $G_{1} \in \mathcal{R}_{P}$. It remains to show that $G_{1} \in \mathcal{R}^{A}$ or, equivalently, that $U^{*} G_{1} \in \mathcal{P}\left(A, \mathcal{R}\left(U^{*} T\right)\right)$ (see Theorem 4.6). A simple computation shows that $\left(U^{*} G_{1}\right)^{2}=U^{*} G_{1}$ and $\mathcal{R}\left(U^{*} G_{1}\right)=\mathcal{R}\left(U^{*} T\right)$, so we only need to prove that $A U^{*} G_{1}$ is selfadjoint. Now, as $G_{1} U^{*}=P_{U A U^{*}, \mathcal{R}(T)}$ then $U A U^{*} G_{1} U^{*}$ is selfadjoint. That is, $U A U^{*} G_{1} U^{*}=U\left(A U^{*} G_{1}\right)^{*} U^{*}$. But, as $\mathcal{R}\left(A U^{*} G_{1}\right)=\mathcal{R}\left(A U^{*} T\right) \subseteq \mathcal{M}$, $\mathcal{R}\left(\left(A U^{*} G_{1}\right)^{*}\right) \subseteq \mathcal{R}\left(G_{1}^{*}\right) \subseteq \mathcal{M}$ and $\mathcal{M} \cap \mathcal{N}(U)=\{0\}$ then $A U^{*} G_{1}=$ $\left(A U^{*} G_{1}\right)^{*}$, as desired. Therefore, $G_{1} \in \mathcal{R}^{A} \cap \mathcal{R}_{P}$ and $G_{1} U^{*}=P_{U A U^{*}, \mathcal{R}(T)}$.

Finally, by Proposition 2.5 and Theorem 4.6, we get that $\left\|G_{1} U^{*}\right\| \leq$ $\left\|G U^{*}\right\|$ for all $G \in \mathcal{R}^{A}$.

The following remark follows from the proof of Theorem 4.9. 
Remark 4.11. Under the hypotheses of Theorem 4.9, for every closed subspace $\mathcal{M}$ of $\mathcal{H}$ such that $\mathcal{M}+\mathcal{N}(U)=\mathcal{H}$ and $R\left(A U^{*} T\right) \subseteq \mathcal{M}$, and every $F \in \mathcal{L}(\mathcal{H})$ such that $U F=P_{U A U^{*}, R(T)}^{*}$ the operator

$$
G_{1}=P_{T} F^{*} Q_{\mathcal{M} / / \mathcal{N}(U)}^{*}
$$

verifies that $G_{1} \in \mathcal{R}^{A} \cap \mathcal{R}_{P}, G_{1} U^{*}=P_{U A U^{*}, R(T)}$ and $\left\|G_{1} U^{*}\right\| \leq\left\|G U^{*}\right\|$ for all $G \in \mathcal{R}^{A}$.

In the next example, we present a family of operators $A \in \mathcal{L}^{+}\left(\ell^{2}\right)$ for which the conditions of Theorem 4.9 hold:

Example 4.12. Suppose that $c_{0}\left(\mathcal{R}(T), \mathcal{N}\left(U^{*}\right)\right)<1$ and $\mathcal{S}=\mathcal{R}(T) \cap \mathcal{R}(U) \neq$ $\{0\}$. Consider $A \in \mathcal{L}^{+}\left(\ell^{2}\right)$ such that $\mathcal{N}(A)=U^{*} \mathcal{S}$ and $\mathcal{R}(A)$ is closed (for instance, $A=I-P_{U^{*} \mathcal{S}}$ satisfies these conditions). Now, it is easy to check that $\mathcal{N}\left(A U^{*}\right)=\mathcal{S}+\mathcal{N}\left(U^{*}\right)$. Then it holds that $\mathcal{N}\left(A U^{*}\right) \cap \mathcal{R}(T) \subseteq$ $\mathcal{R}(U)$. In fact, if $x \in \mathcal{N}\left(A U^{*}\right) \cap \mathcal{R}(T)$ then $x=s+y$, where $s \in \mathcal{S}$, $y \in \mathcal{N}\left(U^{*}\right)$. Thus $x-s \in \mathcal{R}(T) \cap \mathcal{N}\left(U^{*}\right)=\{0\}$. So that $x \in \mathcal{S} \subseteq \mathcal{R}(U)$, i.e., $\mathcal{N}\left(A U^{*}\right) \cap \mathcal{R}(T) \subseteq \mathcal{R}(U)$. Moreover, $\left(A, \mathcal{R}\left(U^{*} T\right)\right)$ is compatible because $\mathcal{R}\left(U^{*} T\right)+\mathcal{N}(A)=\mathcal{R}\left(U^{*} T\right)$ is closed (see Lemma 2.6). Furthermore, as $\mathcal{N}(A) \cap \mathcal{R}\left(U^{*} T\right)=U^{*} \mathcal{S} \neq\{0\}$ then the set $\mathcal{P}\left(A, \mathcal{R}\left(U^{*} T\right)\right)$ is not singleton.

For instance, returning to the Example 4.7 we note that the hypotheses of Theorem 4.9 are verified. Thus, the elements in $\mathcal{R}^{A} \cap \mathcal{R}_{P}$ with the smallest quasi-optimality constant between all $G \in \mathcal{R}^{A}$ are $P_{T} F^{*} Q_{\mathcal{M} / / \mathcal{N}(U)}^{*}=$ $P_{T} F^{*} U^{*}\left(U^{*}\right)^{\dagger} Q_{\mathcal{R}\left(U^{*}\right) / / \mathcal{M}^{\perp}}=P_{U A U^{*}, \mathcal{R}(T)}\left(U^{*}\right)^{\dagger} Q_{\mathcal{R}\left(U^{*}\right) / / \mathcal{M}^{\perp}}$, for every closed subspace $\mathcal{M}$ of $\mathcal{H}$ such that $\mathcal{M}+\mathcal{N}(U)=\mathcal{H}$ and $R\left(A U^{*} T\right) \subseteq \mathcal{M}$. Therefore, since $\mathcal{N}\left(P_{U A U^{*}, \mathcal{R}(T)}\right)=\mathcal{R}\left(U A U^{*} T\right)^{\perp} \cap \mathcal{S}^{\perp}$ we get that $P_{U A U^{*}, \mathcal{R}(T)}=$ $Q_{\mathcal{R}(T) / / \mathcal{R}\left(U A U^{*} T\right)^{\perp} \cap \mathcal{S}^{\perp}}$. Hence, the desired reconstruction operators are fully described.

In Corollary 3.8 we described the set of perfect reconstruction operators with minimal quasi-optimality constant, namely, $\mathcal{R}_{P}\left(Q_{0}\right)$, where $Q_{0}:=$

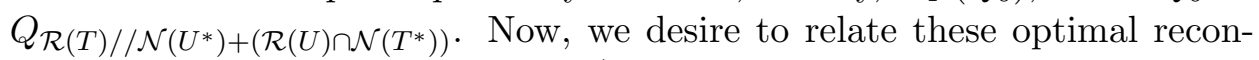
struction operators in $\mathcal{R}_{P}$ with $\mathcal{R}^{A}$ for convenient $A \in \mathcal{L}^{+}\left(\ell^{2}\right)$. In order to simplify the analysis, we shall focus on the operator $G_{0}:=Q_{0}\left(U^{*}\right)^{\dagger} \in$ $\mathcal{R}_{P}\left(Q_{0}\right)$. Therefore, our goal is to provide a family of operators $A \in \mathcal{L}^{+}\left(\ell^{2}\right)$ for which $G_{0} \in \mathcal{R}^{A}$. For this purpose, we introduce the next result due to Corach and Maestrpieri [19, Theorem 5.1]:

Theorem 4.13. Given $E \in \mathcal{Q}$ and $A \in \mathcal{L}^{+}(\mathcal{H})$ the following conditions are equivalent: 
a) $A E=E^{*} A$;

b) $A=A_{1}+A_{2}$ for some $A_{1}, A_{2} \in \mathcal{L}^{+}(\mathcal{H})$ with $\mathcal{R}\left(A_{1}\right) \subseteq \mathcal{R}\left(E^{*}\right)$ and $\mathcal{R}\left(A_{2}\right) \subseteq \mathcal{N}\left(E^{*}\right)$

By means of the above result we characterize the set $\mathcal{B}:=\left\{B \in \mathcal{L}^{+}(\mathcal{H})\right.$ : $\mathcal{R}(B)=\mathcal{R}(U)$ and $\left.Q_{0} \in \mathcal{P}(B, \mathcal{R}(T))\right\}$ as follows:

Corollary 4.14. $\mathcal{B}=\left\{B_{1}+B_{2}: B_{i} \in \mathcal{L}^{+}(\mathcal{H}), \mathcal{R}\left(B_{1}\right)=\mathcal{R}(U) \cap(\mathcal{R}(U) \cap\right.$ $\left.\mathcal{N}\left(T^{*}\right)\right)^{\perp}$ and $\left.\mathcal{R}\left(B_{2}\right)=\mathcal{R}(U) \cap \mathcal{N}\left(T^{*}\right)\right\}$.

Proof. The result follows by Theorem 4.13 and by the fact that if $B_{1}, B_{2} \in$ $\mathcal{L}^{+}(\mathcal{H})$ are such that $B=B_{1}+B_{2}$ has closed range then $R(B)=R\left(B_{1}\right)+$ $R\left(B_{2}\right)$ (see [6, Theorem 3.3]).

Proposition 4.15. Let $\mathcal{A}=\left\{U^{\dagger} B\left(U^{*}\right)^{\dagger}: B \in \mathcal{B}\right\}$. Then, $G_{0}:=Q_{0}\left(U^{*}\right)^{\dagger} \in$ $\mathcal{R}^{A}$ for all $A \in \mathcal{A}$.

Proof. By Theorem 4.6, it suffices to prove that $U^{*} G_{0} \in \mathcal{P}\left(A, \mathcal{R}\left(U^{*} T\right)\right)$. Since $G_{0} \in \mathcal{R}_{P}, U^{*} G_{0} \in \mathcal{Q}$ and $\mathcal{R}\left(U^{*} G_{0}\right)=\mathcal{R}\left(U^{*} T\right)$. Now, for all $A \in \mathcal{A}$ it holds $A U^{*} G_{0}=U^{\dagger} B\left(U^{*}\right)^{\dagger} U^{*} Q_{0}\left(U^{*}\right)^{\dagger}=U^{\dagger} B Q_{0}\left(U^{*}\right)^{\dagger}$ which is a selfadjoint operator because $B \in \mathcal{B}$, i.e., $U^{*} G_{0}$ is $A$-selfadjoint. The proof is complete.

The following result can be found in [8, Theorem 3.3]:

Corollary 4.16. Let $A=\left(U^{*} U\right)^{\dagger}$. Then, $G_{0}:=Q_{0}\left(U^{*}\right)^{\dagger} \in \mathcal{R}^{A}$

Proof. First observe that $P_{U} \in \mathcal{B}$. In fact, $P_{U}=P_{\mathcal{R}(U) \cap\left(\mathcal{R}(U) \cap \mathcal{N}\left(T^{*}\right)\right)^{\perp}}+$ $P_{\mathcal{R}(U) \cap \mathcal{N}\left(T^{*}\right)}$, then, by Corollary 4.14, $P_{U} \in \mathcal{B}$. Now, $A=U^{\dagger}\left(U^{*}\right)^{\dagger}=$ $U^{\dagger} P_{U}\left(U^{*}\right)^{\dagger}$. Therefore, by Proposition 4.15, $G_{0}:=Q_{0}\left(U^{*}\right)^{\dagger} \in \mathcal{R}^{A}$.

\section{References}

[1] Ben Adcock, Anders C. Hansen, and Clarice Poon, Beyond consistent reconstructions: optimality and sharp bounds for generalized sampling, and application to the uniform resampling problem., SIAM J. Math. Anal. 45 (2013), no. 5, 3132-3167.

[2] Esteban Andruchow, Jorge Antezana, and Gustavo Corach, Sampling formulae and optimal factorizations of projections., Sampl. Theory Signal Image Process. 7 (2008), no. 3, 313-331. 
[3] Jorge Antezana and Gustavo Corach, Sampling theory, oblique projections and a question by Smale and Zhou, Appl. Comput. Harmon. Anal. 21 (2006), no. 2, 245-253.

[4] Jorge Antezana, Gustavo Corach, Mariano Ruiz, and Demetrio Stojanoff, Weighted projections and Riesz frames, Linear Algebra Appl. 402 (2005), 367-389.

[5] M. Laura Arias and Cristian Conde, Generalized inverses and sampling problems, J. Math. Anal. Appl. 398 (2013), no. 2, 744-751.

[6] M. Laura Arias, Gustavo Corach, and M. Celeste Gonzalez, Additivity properties of operator ranges, Linear Algebra Appl. 439 (2013), no. 11, $3581-3590$.

[7] _ Saddle point problems, Bott-Duffin inverses, abstract splines and oblique projections, Linear Algebra Appl. 457 (2014), 61-75.

[8] Peter Berger, Karlheinz Gröchenig, and Gerald Matz, Sampling and reconstruction in distinct subspaces using oblique projections, J. Fourier Anal. Appl. 25 (2019), 1080-112.

[9] Yair Censor and Tommy Elfving, Block-iterative algorithms with diagonally scaled oblique projections for the linear feasibility problem, SIAM J. Matrix Anal. Appl. 24 (2002), no. 1, 40-58.

[10] Iterative algorithms with seminorm-induced oblique projections, Abstr. Appl. Anal. (2003), no. 7, 387-406.

[11] Yair Censor, Dan Gordon, and Rachel Gordon, Component averaging: an efficient iterative parallel algorithm for large and sparse unstructured problems, Parallel Comput. 27 (2001), no. 6, 777-808.

[12] Ole Christensen, Frames and Bases-An Introductory Course, Birkhäuser, Boston, 2008.

[13] Maximiliano Contino, Juan Giribet, and Alejandra Maestripieri, Weighted least squares solutions of the equation $A X B-C=0$, Linear Algebra Appl. 518 (2017), 177-197.

[14] Gustavo Corach, Guillermina Fongi, and Alejandra Maestripieri, Weighted projections into closed subspaces, Stud. Math. 216 (2013), no. 2, 131-148. 
[15] Gustavo Corach, Juan I. Giribet, and Alejandra Maestripieri, Sard's approximation processes and oblique projections, Stud. Math. 194 (2009), no. $1,65-80$.

[16] Gustavo Corach and Alejandra Maestripieri, Redundant decompositions, angles between subspaces and oblique projections, Publ. Mat., Barc. 54 (2010), no. 2, 461-484.

[17] Gustavo Corach, Alejandra Maestripieri, and Demetrio Stojanoff, Generalized Schur complements and oblique projections, Linear Algebra Appl. 341 (2002), no. 1-3, 259-272.

[18] Gustavo Corach and Juan I. Giribet, Oblique projections and sampling problems, Integral Equations Oper. Theory 70 (2011), no. 3, 307-322.

[19] Gustavo Corach and Alejandra Maestripieri, A setting for generalized orthogonalization, Ann. Funct. Anal. AFA 5 (2014), no. 1, 128-142.

[20] Gustavo Corach, Alejandra Maestripieri, and Demetrio Stojanoff, Oblique projections and Schur complements, Acta Sci. Math. 67 (2001), no. 1-2, 337-356.

[21] Gustavo Corach, Alejandra Maestripieri, and Demetrio Stojanoff, A classification of projectors, Topological algebras, their applications, and related topics, Banach Center Publ., 67, Polish Acad. Sci. Inst. Math., Warsaw, 2005, 145-160.

[22] Frank Deutsch, The angle between subspaces of a Hilbert space, Approximation theory, wavelets and applications (Maratea, 1994), NATO Adv. Sci. Inst. Ser. C Math. Phys. Sci., vol. 454, Kluwer Acad. Publ., Dordrecht, 1995, 107-130.

[23] Ronald G. Douglas, On majorization, factorization, and range inclusion of operators on Hilbert space, Proc. Am. Math. Soc. 17 (1966), 413-415.

[24] Yonina C. Eldar, Sampling with arbitrary sampling and reconstruction spaces and oblique dual frame vectors, J. Fourier Anal. Appl. 9 (2003), no. $1,77-96$.

[25] _ Sampling without input constraints: consistent reconstruction in arbitrary spaces, Sampling, wavelets and tomography, Boston: Birkhäuser, 2004, 33-60. 
[26] Yonina C. Eldar and Tobias Werther, General framework for consistent sampling in Hilbert spaces, Int. J. Wavelets Multiresolut. Inf. Process. 3 (2005), no. 4, 497-509.

[27] Israel C. Gohberg and Mark G. Krein, Introduction to the Theory of Linear Nonselfadjoint Operators, Translated from the Russian by A. Feinstein. Translations of Mathematical Monographs, Vol. 18, American Mathematical Society, Providence, R.I., 1969.

[28] Tosio Kato, Perturbation Theory for Linear Operators, Die Grundlehren der mathematischen Wissenschaften, Band 132, SpringerVerlag New York, Inc., New York, 1966.

[29] Sujit Kumar Mitra and C. Radhakrishna Rao, Projections under seminorms and generalized Moore Penrose inverses, Linear Algebra Appl. 9 (1974), 155-167.

[30] Arthur Sard, Approximation and variance, Trans. Amer. Math. Soc. 73 (1952), 428-446.

[31] Steve Smale and Ding-Xuan Zhou, Shannon sampling and function reconstruction from point values, Bull. Am. Math. Soc., New Ser. 41 (2004), no. 3, 279-305.

[32] Wai-Shing Tang, Oblique projections, biorthogonal Riesz bases and multiwavelets in Hilbert spaces, Proc. Am. Math. Soc. 128 (2000), no. 2, 463-473.

[33] Michael Unser and Akram Aldroubi, A general sampling theory for nonideal acquisition devices, IEEE Transactions on Signal Processing 42 (1994), no. 11, 2915-2925.

[34] Jiong Wang and Yibin Zheng, On the convergence of generalized simultaneous iterative reconstruction algorithms, IEEE Trans. Image Process. 16 (2007), no. 1, 1-6.

[35] Robert M. Young, An Introduction to Nonharmonic Fourier Series, first ed., Academic Press, Inc., San Diego, CA, 2001.

1 Instituto Argentino de Matemática "Alberto P. Calderón" (CONICET), Buenos Aires, Argentina

2 Dpto. de Matemática, Facultad de Ingeniería, Universidad de Buenos Aires, Buenos Aires, Argentina.

3 Instituto de Ciencias, Universidad Nacional de General Sarmiento, Los Polvorines, Buenos Aires, Argentina

${ }^{a}$ lauraarias@conicet.gov.ar, ${ }^{b}$ celeste.gonzalez@conicet.gov.ar 